\title{
Recent Single Event Effects Compendium of Candidate Electronics for NASA Space Systems
}

\author{
Martha V. O'Bryan, Kenneth A. LaBel, Jonathan A. Pellish, Jean-Marie Lauenstein, Dakai Chen, \\ Cheryl J. Marshall, Timothy R. Oldham, Hak S. Kim, Anthony M. Phan, Melanie D. Berg, \\ Michael J. Campola, Anthony B. Sanders, Paul W. Marshall, Michael A. Xapsos, \\ Raymond L. Ladbury, David F. Heidel, Kenneth P. Rodbell, Jim W. Swonger, \\ Don Alexander, Michael Gauthier, and Brian Gauthier
}

\begin{abstract}
We present the results of single event effects (SEE) testing and analysis investigating the effects of radiation on electronics. This paper is a summary of test results.
\end{abstract}

Index Terms-Single event effects, spacecraft electronics, digital, linear bipolar, and hybrid devices.

\section{INTRODUCTION}

The performance of electronic devices in a space radiation environment is often limited by its susceptibility to SEE. Interpreting the results of SEE testing of complex devices is quite difficult. Given the rapidly changing nature of both technology and the related SEE issues being discovered, SEE test data is very application specific and adequate understanding of the test conditions is critical [1].

Given this limitation of test data (application-specific), studies discussed herein were undertaken to establish the sensitivities of candidate spacecraft electronics as well as new electronic devices to heavy ion and proton-induced single event upset (SEU), single event latchup (SEL), and single event transients (SET). For total ionizing dose (TID) and displacement damage (DD) results, see a companion paper submitted to the 2011 IEEE NSREC Radiation Effects Data Workshop entitled: "Recent Total Ionizing Dose and

This work was supported in part by the NASA Electronic Parts and Packaging Program (NEPP), NASA Flight Projects, and the Defense Threat Reduction Agency (DTRA) under IACRO\# 10-49771, LACRO\# 11-43951.

Martha V. O'Bryan is with MEl Technologies Inc., work performed for NASA Goddard Space Flight Center (GSFC), Code 561.4, Bldg. 22, Rm. 062A, Greenbelt, MD 20771 (USA), phone: 301-286-1412, fax: 301-2864699, email: martha.v.obryan@nasa.gov.

Kenneth A. LaBel, Jonathan A. Pellish, Jean-Marie Lauenstein, Dakai Chen, Cheryl Marshall, Michael J. Campola, Anthony B. Sanders, Michael A. Xapsos, and Ray L. Ladbury are with NASA/GSFC, Code 561.4, Greenbelt, MD 20771 (USA), email: kenneth.a.label@nasa.gov, jonathan.a.pellish@ nasa.gov, jean.m. lauenstein@ nasa.gov, Dakai.Chen-1@nasa.gov, cheryl.j.marshall@nasa.gov,michael.j.campola@nasa.gov, anthony.b.sanders @nasa.gov, michael.a.xapsos@ nasa.gov, and raymond.I.ladbury@nasa.gov.

Hak S. Kim, Anthony M. Phan, and Melanie Berg are with MEl Technologies, Inc., work performed for NASA/GSFC, Code 561.4, Greenbelt, MD 20771 (USA), email: Hak.S.Kim@nasa.gov, Anthony.M.Phan @nasa.gov, and Melanie.D.Berg@nasa.gov.

Timothy R. Oldham is with Perot Systems Government Services, Inc., work performed for NASA/GSFC, Code 561.4, Greenbelt, MD 20771 (USA), email: timothy.r. oldham@nasa.gov.

Paul Marshall is a Consultant, email: pwmarshall@aol.com.

David F. Heidel, and Kenneth P. Rodbell are with IBM T.J. Watson Research Center.

Jim Swonger, and Don Alexander are with Peregrine Semiconductor.

Michael Gauthier, and Brian Gauthier are with Semicoa.
Displacement Damage Compendium of Candidate Electronics for NASA Space Systems" by D. Cochran, et al. [2].

\section{TEST TECHNIQUES AND SETUP}

\section{A. Test Facilities}

All SEE tests were performed between February 2010 and February 2011. Heavy ion experiments were conducted at Lawrence Berkeley National Laboratory (LBNL) [3], and at Texas A\&M University Cyclotron (TAMU) [4]. Both of these facilities are suitable for providing a variety of ions over a range of energies for testing. The devices under test (DUTs) were irradiated with heavy ions having linear energy transfers (LETs) ranging from 0.59 to $120 \mathrm{MeV} \cdot \mathrm{cm}^{2} / \mathrm{mg}$. Fluxes ranged from $1 \times 10^{2}$ to $1 \times 10^{7}$ particles $/ \mathrm{cm}^{2} / \mathrm{s}$, depending on device sensitivity. Representative ions used are listed in Table I. LETs between the values listed were obtained by changing the angle of incidence of the ion beam with respect to the DUT, thus changing the path length of the ion through the DUT and the "effective LET" of the ion [5]. Energies and LETs available varied slightly from one test date to another.

Proton SEE tests were performed at three facilities: the University of California at Davis (UCD) Crocker Nuclear Laboratory (CNL) [6], the Indiana University Cyclotron Facility (IUCF) [7], and at a $2 \mathrm{MeV}$ Van de Graaff (VdG) particle accelerator. Proton test energies incident on the DUT are listed in Table II.

Laser SEE tests were performed at the pulsed laser facility at the Naval Research Laboratory (NRL) [8] [9]. The laser light had a wavelength of $590 \mathrm{~nm}$ resulting in a skin depth (depth at which the light intensity decreased to $1 / \mathrm{e}$ - or about $37 \%$ - of its intensity at the surface) of $2 \mu \mathrm{m}$. A nominal pulse rate of $1 \mathrm{kHz}$ was utilized. 
TABLE I: HEAVY ION TEST FACILITIES AND TEST HEAVY lONS

\begin{tabular}{|c|c|c|c|c|}
\hline & Ion & $\begin{array}{c}\text { Energy } \\
\text { (MeV) }\end{array}$ & $\begin{array}{c}\text { Surface } \\
\text { LET in Si } \\
\left.\text { (MeV·cm }{ }^{2} / \mathrm{mg}\right) \\
\text { (Normal Incidence) }\end{array}$ & $\begin{array}{l}\text { Range in } \\
\text { Si ( } \mu \mathrm{m})\end{array}$ \\
\hline \multirow[t]{7}{*}{ LBNL } & ${ }^{18} \mathrm{O}$ & 184 & 2.2 & 227 \\
\hline & ${ }^{22} \mathrm{Ne}$ & 216 & 3.5 & 175 \\
\hline & ${ }^{40} \mathrm{Ar}$ & 400 & 9.7 & 130 \\
\hline & ${ }^{65} \mathrm{Cu}$ & 659 & 21 & 110 \\
\hline & ${ }^{80} \mathrm{Kr}$ & 886 & 31 & 110 \\
\hline & ${ }^{136} \mathrm{Xe}$ & 1330 & 59 & 97 \\
\hline & \multicolumn{4}{|c|}{$10 \mathrm{MeV}$ per AMU tune } \\
\hline \multirow{12}{*}{ TAMU } & ${ }^{20} \mathrm{Ne}$ & 300 & 2.5 & 316 \\
\hline & ${ }^{40} \mathrm{Ar}$ & 599 & 7.7 & 229 \\
\hline & ${ }^{63} \mathrm{Cu}$ & 944 & 17.8 & 172 \\
\hline & ${ }^{84} \mathrm{Kr}$ & 1259 & 25.4 & 170 \\
\hline & ${ }^{109} \mathrm{Ag}$ & 1634 & 38.5 & 156 \\
\hline & ${ }^{129} \mathrm{Xe}$ & 1934 & 47.3 & 156 \\
\hline & \multicolumn{4}{|c|}{$15 \mathrm{MeV}$ per AMU tune } \\
\hline & ${ }^{22} \mathrm{Ne}$ & 545 & 1.8 & 799 \\
\hline & ${ }^{40} \mathrm{Ar}$ & 991 & 5.5 & 493 \\
\hline & ${ }^{84} \mathrm{Kr}$ & 2081 & $\cdot 19.8$ & 332 \\
\hline & ${ }^{130} \mathrm{Xe}$ & 3197 & 38.9 & 286 \\
\hline & \multicolumn{4}{|c|}{$25 \mathrm{MeV}$ per AMU tune } \\
\hline
\end{tabular}

TABLE II: PROTON TEST FACILITIES

University of California at Davis (UCD) Crocker Nuclear Laboratory $(\mathrm{CNL})$, energy tunes ranged from 6.5 to $63 \mathrm{MeV}$, flux ranged from $8 \times 10^{7}$ to $1 \times 10^{9}$ particles $/ \mathrm{cm}^{2} / \mathrm{s}$.

Indiana University Cyclotron Facility (IUCF), energy ranged from 63 to $198 \mathrm{MeV}$, flux ranged from $5 \times 10^{5}$ to $3 \times 10^{9}$ particles $/ \mathrm{cm}^{2} / \mathrm{s}$.

TABLE III: LASER TEST FACILITY

Naval Research Laboratory (NRL) Pulsed Laser SEE Test Facility Laser: $590 \mathrm{~nm}, 1$ ps pulse width, beam spot size $\sim 1.2 \mu \mathrm{m}$

\section{B. Test Method}

Unless otherwise noted, all tests were performed at room temperature and with nominal power supply voltages. We recognize that high-temperature and worst-case power supply conditions are recommended for single event latchup (SEL) device qualification.

1) SEE Testing - Heavy Ion:

Depending on the DUT and the test objectives, one or more of three SEE test methods were typically used:

Dynamic - the DUT was exercised continually while being exposed to the beam. The events and/or bit errors were counted, generally by comparing the DUT output to an unirradiated reference device or other expected output (Golden chip or virtual Golden chip methods) [10]. In some cases, the effects of clock speed or device operating modes were investigated. Results of such tests should be applied with caution due to the application-specific nature of the results.

Static - the DUT was loaded prior to irradiation; data were retrieved and errors were counted after irradiation.
Biased - the DUT was biased and clocked while powér consumption was monitored for SEL or other destructive effects. In most SEL tests, functionality was also monitored.

In SEE experiments, DUTs were monitored for soft errors, such as SEUs and for hard errors, such as single event gate rupture (SEGR). Detailed descriptions of the types of errors observed are noted in the individual test reports [11],[12].

SET testing was performed using a high-speed oscilloscope controlled via Labview® Individual criteria for SETs are specific to the device being tested and application. Please see the individual test reports for details [11].

Heavy ion SEE sensitivity experiments include measurement of the Linear Energy Transfer threshold (LET $T_{t h}$ ) and cross section at the maximum measured LET. The LET th is defined as the maximum LET value at which no effect was observed at an effective fluence of $1 \times 10^{7}$ particles $/ \mathrm{cm}^{2}$. In the case where events are observed at the smallest LET tested, LET $_{\text {th }}$ will either be reported as less than the lowest measured LET or determined approximately as the LET $_{\text {th }}$ parameter from a Weibull fit. In the case of SEGR experiments, measurements are made of the SEGR threshold $V_{d s}$ as a function of LET at a fixed $\mathrm{V}_{\mathrm{gs}}$.

\section{2) SEE Testing - Proton}

Proton SEE tests were performed in a manner similar to heavy ion exposures. However, because protons cause SEE via indirect ionization of recoil particles, results are parameterized in terms of proton energy rather than LET. Because such proton-induced nuclear interactions are rare, proton tests also feature higher cumulative fluences and particle flux rates than heavy ion experiments.

\section{3) Pulsed Laser Facility Testing}

The DUT was mounted on an X-Y-Z stage in front of a $100 \mathrm{x}$ lens that produced a spot diameter of about $1.2 \mu \mathrm{m}$ at full-width half-maximum (FWHM). The X-Y-Z stage can be moved in steps of $0.1 \mu \mathrm{m}$ for accurate positioning of SEU sensitive regions in front of the focused beam. An illuminator together with a charge coupled device camera and monitor were used to image the area of interest, thereby facilitating accurate positioning of the device in the beam. The pulse energy was varied in a continuous manner using a polarizer/half-waveplate combination and the energy was monitored by splitting off a portion of the beam and directing it at a calibrated energy meter.

\section{TEST RESUltS OVERVIEW}

Abbreviations and conventions are listed in Table IV. Abbreviations for principal investigators (PIs) are listed in Table V, and SEE results are summarized in Table VI. Unless otherwise noted, all LETs are in $\mathrm{MeV} \cdot \mathrm{cm}^{2} / \mathrm{mg}$ and all cross sections are in $\mathrm{cm}^{2} /$ device. All SEL tests are performed at a fluence of $1 \times 10^{-7}$ particles $/ \mathrm{cm}^{2}$ unless otherwise noted. 
TABLE IV: ABBREVIATIONS AND CONVENTIONS

LET $=$ linear energy transfer $\left(\mathrm{MeV} \cdot \mathrm{cm}^{2} / \mathrm{mg}\right)$

$\mathrm{LET}_{\text {th }}=$ linear energy transfer threshold (the maximum LET value at which no effect was observed at an effective fluence of $1 \times 10^{7}$ particles $/ \mathrm{cm}^{2}-$ in $\mathrm{MeV} \cdot \mathrm{cm}^{2} / \mathrm{mg}$ )

$<=$ SEE observed at lowest tested LET

$>=$ no SEE observed at highest tested LET

$\sigma=$ cross section $\left(\mathrm{cm}^{2} /\right.$ device, unless specified as $\left.\mathrm{cm}^{2} / \mathrm{bit}\right)$

$\sigma_{\text {max measured }}=$ cross section at maximum measured LET ( $\mathrm{cm}^{2} /$ device, unless specified as $\mathrm{cm}^{2} / \mathrm{bit}$ )

$\mathrm{ADC}=$ analog to digital converter

App. Spec. $=$ application specific

ASET $=$ analog single-event transient

BiCMOS = bipolar complementary metal oxide semiconductor

CMOS = complementary metal oxide semiconductor

$\mathrm{DAC}=$ digital to analog converter

DTMR $=$ distributed triple modular redundancy

DUT $=$ device under test

EDAC $=$ error detection and correction

$\mathrm{FPGA}=$ field programmable gate array

$\mathrm{GaAs}=$ gallium arsenide

$\mathrm{H}=$ heavy ion test

In $\mathrm{GaP}=$ indium gallium phosphide

$\mathrm{L}=$ laser test

LBNL = Lawrence Berkeley National Laboratory

LCDT $=$ low cost digital tester

$\mathrm{LC}^{2} \mathrm{MOS}=$ linear compatible CMOS $\left(\mathrm{LC}^{2} \mathrm{MOS}\right)$ process

LDC $=$ lot date code

$\mathrm{LO}=$ local oscillator

LTMR $=$ localized triple modular redundancy

MDAC = multiplying digital-to-analog converter

MESFET $=$ metal semiconductor field effect transistor

MMIC = microwave monolithic integrated circuit

MOSFET $=$ metal oxide semiconductor field effect transistor

MSOP $=$ mini small outline package

$\mathrm{NA}=$ not available

NRL $=$ Naval Research Laboratory

$\mathrm{P}=$ proton test $(\mathrm{SEE})$
$\mathrm{PCM}=$ phase change memory

Table IV: Abbreviations and Conventions (Cont.)

$\mathrm{PI}=$ principal investigator

$\mathrm{PN}=$ part number

$\mathrm{POL}=$ point of load

SEB $=$ single event burnout

$\mathrm{SEE}=$ single event effect

SEFI = single event functional interrupt

SEGR $=$ single event gate rupture

$\mathrm{SEL}=$ single event latchup

SET $=$ single event transient

SEU $=$ single event upset

$\mathrm{SiGe}=$ silicon germanium

$\mathrm{VdG}=$ Van de Graaff

VDMOS $=$ drain voltage MOSFET

$\mathrm{V}_{\mathrm{ds}}=$ drain-source voltage

$\mathrm{V}_{\mathrm{gs}}=$ gate-source voltage

$\mathrm{V}_{\mathrm{th}}^{\mathrm{gs}}=$ gate threshold voltage

WC $=$ worst case

TABLE V: LIST OF PRINCIPAL INVESTIGATORS

\begin{tabular}{lc}
\hline \multicolumn{1}{c}{ Principal Investigator (PI) } & Abbreviation \\
\hline Melanie Berg & MB \\
Michael Campola & MiC \\
Dakai Chen & DC \\
Hak Kim & HK \\
Jean-Marie Lauenstein & JML \\
Timothy Oldham & TO \\
Jonathan Pellish & JP \\
Anthony (Tony) Sanders & AS \\
Michael Xapsos & MX \\
\hline
\end{tabular}

TABLE VI: SUMMARY OF SEE TEST RESULTS

\begin{tabular}{|c|c|c|c|c|c|c|c|c|c|}
\hline Part Number & Manufacturer & LDC & $\begin{array}{l}\text { Device } \\
\text { Function }\end{array}$ & $\begin{array}{l}\text { Tech- } \\
\text { nology }\end{array}$ & $\begin{array}{l}\text { Particle: } \\
\text { (Facility/Date) P.I. }\end{array}$ & $\begin{array}{l}\text { Test Results LET in } \\
\mathrm{MeV} \cdot \mathrm{cm}^{2} / \mathrm{mg} \sigma \text { in } \\
\mathrm{cm}^{2} / \text { device, unless } \\
\text { otherwise specified }\end{array}$ & 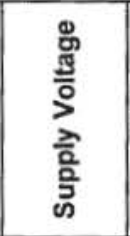 & 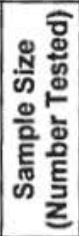 & 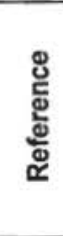 \\
\hline \multicolumn{10}{|l|}{ ADC/DAC: } \\
\hline AD7847 & Analog Devices & $0801 \mathrm{~A}$ & DAC & BiCMOS & H: (TAMU1ODEC) JP & H: SEL LET $T_{m}>82 @ 65^{\circ} \mathrm{C}$ & $+1-15 \mathrm{~V}$ & 3 & [13] \\
\hline ADS7881 & \begin{tabular}{|c|}
$\begin{array}{c}\text { Texas } \\
\text { Instruments }\end{array}$ \\
\end{tabular} & 1010 & $A D C$ & CMOS & $\mathrm{H}:($ TAMU10OCT) JP & H: SEL LET th $>77 @ 65^{\circ} \mathrm{C}$ & $+1-5 \mathrm{~V}$ & 4 & [14] \\
\hline LTC1864L & $\begin{array}{l}\text { Linear } \\
\text { Technology }\end{array}$ & $9 D 17$ & 16-bit ADC & $\begin{array}{l}\text { MSOP } \\
\text { CMOS }\end{array}$ & $\mathrm{H}:$ (TAMU10AUG) MB & $\begin{array}{l}\text { H: SEL LET } \\
1.0<\text { SEU LET }\end{array}$ & \begin{tabular}{|c|}
$3.3 \mathrm{~V} \mathrm{~V} / \mathrm{O}$ \\
$1.5 \mathrm{~V}$ \\
Core \\
\end{tabular} & 3 & [15] \\
\hline $\begin{array}{l}\text { AD7872 } \\
\text { (Generic PN); } \\
\text { 7872ARPFS } \\
\text { (Package PN) }\end{array}$ & $\begin{array}{c}\text { Analog Devices } \\
\text { (Maxwell } \\
\text { Package) }\end{array}$ & 1008 & 14-bit ADC & LC ${ }^{2} M O S$ & $\begin{array}{l}\text { H: (TAMU10FEB) } \\
\text { MiC }\end{array}$ & $\begin{array}{l}\text { H: SEL LET } T_{\text {th }}>59.2 @ 70^{\circ} \mathrm{C} ; \\
\text { SEU LET } \\
\text { SET LET }<8.8 ; \\
\text { Transient pulse amplitude } \\
>10 \mathrm{mV} \text {. }\end{array}$ & $5 \mathrm{~V}$ & 3 & [16] \\
\hline \multicolumn{10}{|c|}{ DC-DC Converter/POL: } \\
\hline PE9915X & $\begin{array}{c}\text { Peregrine } \\
\text { Semiconductor }\end{array}$ & $\begin{array}{l}\text { S11060-2; } \\
\text { S11058-2 }\end{array}$ & $\begin{array}{l}\text { Step down } \\
\text { switching } \\
\text { regulator }\end{array}$ & $\begin{array}{l}\text { CMOS } \\
\text { SOS }\end{array}$ & H: (LBNL11JAN) DC & $\begin{array}{l}\text { H: SELSEGR/SEB } \\
\text { LET th }>118 \text { @ room } \\
\text { temperature. }\end{array}$ & $5 \mathrm{~V}$ & 3 & [17] \\
\hline MSK5059RH & M. S. Kennedy & \begin{tabular}{|c|} 
MSK \\
$5059 R H G$ \\
Be0 \\
51651 \\
USA \\
\end{tabular} & $\begin{array}{l}\text { Step down } \\
\text { switching } \\
\text { regulator }\end{array}$ & BiCMOS & $\begin{array}{l}\text { H: (TAMU10DEC; } \\
\text { TAMU11JUN) DC }\end{array}$ & $\begin{array}{l}\text { H: SEL/SEGR/SEB } \\
\text { LET }_{\text {th }}>87.5 \text { @ room } \\
\text { temperature; } \\
\text { Further tests pending. }\end{array}$ & $7 \mathrm{~V}$ & 3 & [18] \\
\hline MSK5820 & $\begin{array}{c}\text { Linear } \\
\text { Technology }\end{array}$ & 1014 & $\begin{array}{l}\text { Linear } \\
\text { Voltage } \\
\text { Regulator }\end{array}$ & Hybrid & $\begin{array}{l}\text { H: (TAMU10MAY) } \\
\text { MiC }\end{array}$ & $\begin{array}{l}\text { H: SET LET } \\
\text { Typically SET } \sim 70 \text {; } \\
\text { WC transient } \sim 180 \mathrm{us}, 100 \mathrm{mV} \text {. } \\
\text { WC }\end{array}$ & $5 \mathrm{~V}$ & 2 & [19] \\
\hline SMRT28515T & Crane & 0824 & $\begin{array}{l}\text { DC-DC } \\
\text { Converter }\end{array}$ & Hybrid & $\begin{array}{l}\text { H: (TAMU1OMAR; } \\
\text { LBNL10JUN; } \\
\text { TAMU1ONOV) AS }\end{array}$ & $\begin{array}{l}\text { H: SEL LET } \\
\text { SET LET }>86.3 ; \\
\text { SETs observed at } \\
\sim+1-300 \text { mV peak, } 600 \text { ns. }\end{array}$ & $+l-15 \mathrm{~V}$ & 1 & [20] \\
\hline
\end{tabular}

To be presented by Martha O'Bryan at the International Electrical and Electronics Engineering (IEEE) Nuclear and Space Radiation Effects Conference (NSREC), July 27, 2011, Las Vegas, NV, and published on nepp.nasa.gov and radhome.gsfc.nasa.gov. 


\begin{tabular}{|c|c|c|c|c|c|c|c|c|c|}
\hline Part Number & Manufacturer & LDC & $\begin{array}{l}\text { Device } \\
\text { Function }\end{array}$ & $\begin{array}{l}\text { Tech- } \\
\text { nology }\end{array}$ & $\begin{array}{c}\text { Particle: } \\
\text { (Facility/Date) P.I. }\end{array}$ & $\begin{array}{l}\text { Test Results LET in } \\
\mathrm{MeV} \cdot \mathrm{cm}^{2} / \mathrm{mg} \sigma \text { in } \\
\mathrm{cm}^{2} / \text { device, unless } \\
\text { otherwise specified }\end{array}$ & 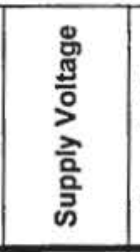 & 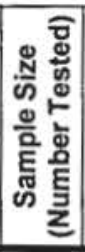 & 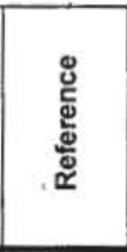 \\
\hline SMTR283R3S & Crane & $\begin{array}{l}0733 ; \\
0408 ; \\
0722\end{array}$ & $\begin{array}{c}D C-D C \\
\text { Converter }\end{array}$ & Hybrid & $\begin{array}{l}\text { H: (TAMU10MAR; } \\
\text { TAMU10NOV) AS }\end{array}$ & $\begin{array}{l}\text { H: SEL LET in }>86.3 ; \\
\text { SET LET } \\
\text { SETs observed at } \sim 700 \mathrm{mV} \\
\text { peak, 100us. }\end{array}$ & $3.3 \mathrm{~V}$ & 3 & [21] [22] \\
\hline SMSA2812D & Crane & 0733 & $\begin{array}{c}\text { Dual DC-DC } \\
\text { Converter }\end{array}$ & Hybrid & $\begin{array}{l}\text { H: (TAMU10MAR; } \\
\text { TAMU1ONOV) AS }\end{array}$ & $\begin{array}{l}\text { H: SEL LET in }>86.3 ; \\
\text { SET LET th }<28.8 ; \\
\text { SETs observed at } \sim+1-300 \mathrm{mV} \\
\text { peak to peak, } 700 \text { us; }\end{array}$ & $+/-12 \mathrm{~V}$ & 2 & [23] [24] \\
\hline MFP0507S & Crane & \begin{tabular}{|c|} 
LDC Not \\
Released; \\
markings \\
$0077 ;$ \\
$0078 ;$ \\
$0082 ;$ \\
0083 \\
\end{tabular} & $\begin{array}{c}\text { DC-DC } \\
\text { Converter }\end{array}$ & Hybrid & $\begin{array}{l}\text { H: (TAMU10DEC; } \\
\text { LBNL11JAN) AS }\end{array}$ & $\begin{array}{l}\text { H: SEL LET } \text { Lit }>85.4 ; \\
\text { SET LET }<3.49 ; \\
\text { SETs at } 0.8 \mathrm{~V} \text { were } \sim 200 \mathrm{mV} \text {, } \\
100 \text { us; at } 3.3 \mathrm{~V} \text { SETs were } \\
<800 \mathrm{mV}, 200 \text { us. }\end{array}$ & $.8 \mathrm{~V} ; 3 / 3 \mathrm{~V}$ & 4 & [25] [26] \\
\hline SLH2812D & Crane & 0748 & $\begin{array}{c}\text { Dual DC-DC } \\
\text { Converter }\end{array}$ & Hybrid & $\begin{array}{l}\text { H: (LBNL10JUN; } \\
\text { TAMU10NOV; } \\
\text { TAMU10MAR) AS }\end{array}$ & $\begin{array}{l}\text { H: SEL LET } T_{\text {th }}>86.3 ; \\
\text { SET LET } \\
\text { SETs observed at } \sim 1 \mathrm{~V} \text { peak, } \\
700 \text { ns. }\end{array}$ & $+l-12 \mathrm{~V}$ & 3 & [27] [28] \\
\hline SMFLHP2815S & Crane & 0623 & $\begin{array}{c}\text { DC-DC } \\
\text { Converter }\end{array}$ & Hybrid & $\begin{array}{l}\text { H: (TAMU10MAR; } \\
\text { TAMU10NOV) AS }\end{array}$ & $\begin{array}{l}\text { H: SEL LET } T_{\text {th }}>86.3 ; \\
\text { SET LET } \\
\text { SETs observed at } ~ \\
\text { 200us. } \\
\text { 200 peak, }\end{array}$ & $+l-15 \mathrm{~V}$ & 2 & [29] [30] \\
\hline MSK5978RH & M. S. Kennedy & 1028 & $\begin{array}{l}\text { LDO Positive } \\
\text { Adjustable } \\
\text { Voltage } \\
\text { Regulator }\end{array}$ & $\begin{array}{l}\text { Linear } \\
\text { Bipolar }\end{array}$ & L: (NRL100CT) JP & $\begin{array}{l}\text { L: SETs are approximately }+/- \\
150 \mathrm{mV} \text { and } 40 \mu \mathrm{s} \text { worst-case } \\
\text { with a } 0.25 \Omega, 22 \mu \mathrm{F} \text { tantalum } \\
\text { capacitor. } \\
\text { No destructive effects } \\
\text { observed. }\end{array}$ & $\begin{array}{c}16,5.6 \\
5.0,3.9 \\
2.2 \mathrm{~V}\end{array}$ & 2 & [31] \\
\hline S2803R3S & $\begin{array}{l}\text { International } \\
\text { Rectifier }\end{array}$ & 0324 & $\begin{array}{c}\text { DC-DC } \\
\text { Converter }\end{array}$ & Hybrid & L: (NRL10MAY) AS & $\begin{array}{l}\text { L: One photon absorption; } \\
\text { SETs observed at } \\
\sim+1 \text {-150mV, 100us. }\end{array}$ & $3.3 \mathrm{~V}$ & 2 & [32] \\
\hline SMSA2815S & Crane & 0617 & $\begin{array}{c}\text { DC-DC } \\
\text { Converter }\end{array}$ & Hybrid & L: (NRL10MAY) AS & $\begin{array}{l}\text { L: One photon absorption; } \\
\text { SETs observed at } \sim+160 \mathrm{mV} \\
\text { and }-192 \mathrm{mV}, 100 \text { s. }\end{array}$ & $+l-15 \mathrm{~V}$ & 2 & [33] \\
\hline \multicolumn{10}{|l|}{ FPGA: } \\
\hline АЗРЕ3000 & Actel & 0832 & FPGA & cMOS & H: (TAMU10MAY) MB & $\begin{array}{l}\text { H: SEL LET } \text { th }_{\text {th }}>57 \text { @ room } \\
\text { temperature; } \\
\text { No Mitigation and LTMR } \\
1.0<\text { SEU LET }<2.8 ; \\
\text { DTMR: } 8.6<\text { SEULET }<12.0\end{array}$ & $\begin{array}{c}3.3 \mathrm{~V} \mathrm{VO} ; \\
1.5 \mathrm{~V} \\
\text { Core }\end{array}$ & 6 & [34] [35] \\
\hline RTAX2000S & Actel & $\begin{array}{l}\text { 0311; } \\
\text { 0838; } \\
\text { 0526; }\end{array}$ & FPGA & $\begin{array}{c}\text { Antifuse } \\
\text { Tech- } \\
\text { nologyl } \\
\text { CMOS }\end{array}$ & $\begin{array}{l}\text { H: (TAMU10MAY; } \\
\text { TAMU10AUG; } \\
\text { TAMU10DEC) MB }\end{array}$ & 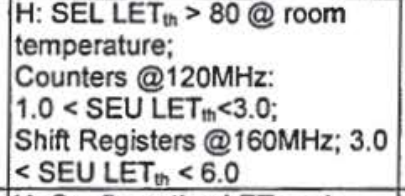 & $\begin{array}{c}2.5 \mathrm{~V} \\
3.3 \mathrm{~V} v \mathrm{i} ; \\
1,5 \mathrm{~V} \\
\text { Core }\end{array}$ & 4 & [35] [36] \\
\hline ACSPD60 & Achronix & 0921 & FPGA & cmos & $\begin{array}{l}\text { H: (TAMU10MAR) } \\
\text { MB; } \\
\text { P (IU10MAR) MB }\end{array}$ & $\begin{array}{l}\text { H: Configuration LET } \text { th }_{\text {th }}<1 ; \\
\text { Tests that contained a large } \\
\text { amount of bit upsets also } \\
\text { experienced sharp current } \\
\text { jumps. This is more than likely } \\
\text { not SEL but many } \\
\text { configuration bits in the wrong } \\
\text { state causing contention within } \\
\text { the fabric. } \\
\text { P: SEU } \sigma=2 \times 10^{-3} \mathrm{~cm}^{2} / \text { device } \\
\text { @ } 45 \mathrm{MeV}\end{array}$ & $\begin{array}{l}3.3 \mathrm{~V} \text { io; } \\
1 \mathrm{~V} \text { Core }\end{array}$ & 1 & [37] [38] \\
\hline XC6SLX16 & Xilin $x$ & 0929 & FPGA & $\begin{array}{l}45 \mathrm{~nm} \\
\mathrm{CMOS}\end{array}$ & P (IU10MAR) MB & $\begin{array}{l}\text { P: No SEL observed at room } \\
\text { temperature proton energy } \\
<200 \mathrm{MeV}_{i} \\
\text { Configuration SEU } \sigma=1 \times 10^{-14} \\
\mathrm{~cm}^{2} / \text { bit @ 63 MeV }\end{array}$ & $\begin{array}{l}2.5 \mathrm{~V} \mathrm{I} O \mathrm{O} \\
1 \mathrm{~V} \text { Core }\end{array}$ & 2 & [39] \\
\hline
\end{tabular}

To be presented by Martha O'Bryan at the International Electrical and Electronics Engineering (IEEE) Nuclear and Space Radiation Effects Conference (NSREC), July 27, 2011, Las Vegas, NV, and published on nepp.nasa.gov and radhome.gsfc.nasa.gov. 


\begin{tabular}{|c|c|c|c|c|c|c|c|c|c|}
\hline Part Number & Manufacturer & LDC & $\begin{array}{l}\text { Device } \\
\text { Function }\end{array}$ & $\begin{array}{l}\text { Tech- } \\
\text { nology }\end{array}$ & $\begin{array}{c}\text { Particle: } \\
\text { (Facility/Date) P.I. }\end{array}$ & $\begin{array}{l}\text { Test Results LET in } \\
\mathrm{MeV} \cdot \mathrm{cm}^{2} / \mathrm{mg} \sigma \text { in } \\
\mathrm{cm}^{2} / \mathrm{device} \text {, unless } \\
\text { otherwise specified }\end{array}$ & 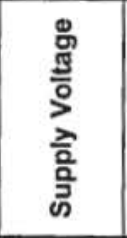 & 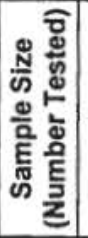 & 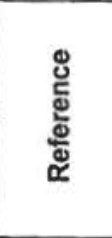 \\
\hline \multicolumn{10}{|c|}{ Linear and Analog Devices: } \\
\hline LTC2054HV & $\begin{array}{c}\text { Linear } \\
\text { Technology }\end{array}$ & $104 \pi T$ & $\begin{array}{c}\text { Operational } \\
\text { Amplifier }\end{array}$ & CMOS & H: (TAMU10OCT) JP & H: SEL LET th > $77 @ 65^{\circ} \mathrm{C}$ & $+1.5 \mathrm{~V}$ & 4 & [40] \\
\hline HS9-1840ARH & Intersil & 0902 & $\begin{array}{l}\text { 16-channel } \\
\text { Analog } \\
\text { Multiplexer }\end{array}$ & CMOS & L: (NRL1OMAY) MiC & $\begin{array}{l}\text { L: SETs typically under } \\
\sim 100 \mathrm{mV} \\
\text { WC transient } 250 \mathrm{mV} \text {. }\end{array}$ & $5 \mathrm{~V}$ & 2 & [41] \\
\hline ISL28196 & Intersil & 0724 & $\begin{array}{l}\text { Voltage } \\
\text { comparator }\end{array}$ & CMOS & L: (NRL10MAR) DC & $\begin{array}{l}\text { L: Laser tested at } 25^{\circ} \mathrm{C} \text { to } \\
75^{\circ} \mathrm{C} \text {. Maximum amplitude } \\
\text { exceeds } 5 \mathrm{~V} \text { (saturated by } \\
\text { supply limits) with full width } \\
\text { half max pulse width of } \sim 10 \\
\mu \text { s. SETs decreased in } \\
\text { magnitude with increasing } \\
\text { temperature. }\end{array}$ & $5 \mathrm{~V}$ & 5 & [42] \\
\hline LTC6400-20 & $\begin{array}{l}\text { Linear } \\
\text { Technology }\end{array}$ & $\begin{array}{l}0746 ; \\
0705\end{array}$ & $\begin{array}{l}\text { Differential } \\
\text { Amplifier }\end{array}$ & $\mid \begin{array}{c}\text { SiGe } \\
\text { BiCMOS }\end{array}$ & $\begin{array}{l} \\
\text { L: (NRL10MAR) DC; } \\
\text { P (IU10MAR) DC/JP }\end{array}$ & $\begin{array}{l}\text { L: Operating at } 500 \mathrm{MHz} \text {. The } \\
\text { SETs distort the sinusoidal } \\
\text { signal for } 1 \text { to } 2 \text { clock periods. } \\
\text { SETs are less significant than } \\
\text { those from heavy-ion test. TID- } \\
\text { irradiated part showed higher } \\
\text { sensitivity than the virgin part. } \\
\text { P: Operating at } 200 \mathrm{MHz} \text {. } 200 \\
\text { MeV protons caused small } \\
\text { distortions to the sinusoidal } \\
\text { signal, affecting } 1 / 2 \text { clock } \\
\text { period. Changes in the SET } \sigma \\
\text { with fluence likely due to } \\
\text { deviations from a Poisson } \\
\text { distribution and/or part-to-part } \\
\text { variation. }\end{array}$ & $3 \mathrm{~V}$ & 2 & [43] [44] \\
\hline & \multicolumn{9}{|c|}{ Power MOSFET/Transistor: } \\
\hline SiB455EDK & $\begin{array}{c}\text { Vishay } \\
\text { Intertechnology }\end{array}$ & $9 Q Z$ & $\begin{array}{l}12 \text { V p-type } \\
\text { Power } \\
\text { MOSFET }\end{array}$ & Trench & $\begin{array}{l}\text { H: (TAMU10AUG) } \\
\text { JML }\end{array}$ & 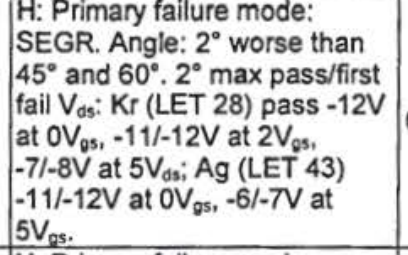 & $0,2,5 \mathrm{~V}_{0 \mathrm{~s}}$ & 20 & [45] \\
\hline Si7431dp & $\begin{array}{c}\text { Vishay } \\
\text { Intertechnology }\end{array}$ & $\begin{array}{c}77 \\
\text { W54B } \\
\& \\
A B \\
\text { W08B }\end{array}$ & $\begin{array}{c}200 \text { V p-type } \\
\text { Power } \\
\text { MOSFET }\end{array}$ & Trench & $\begin{array}{l}\text { H: (LBNL11JAN, } \\
\text { LBNL11MAR) JML }\end{array}$ & 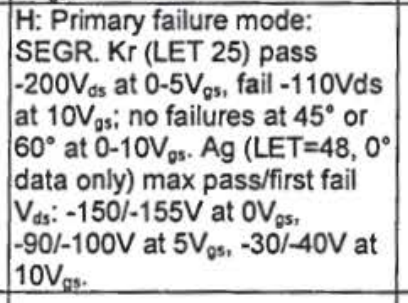 & $\begin{array}{l}0,5 \\
10 \mathrm{~V}_{0 s} \\
\end{array}$ & 22 & [46] \\
\hline SCF9550 & Semicoa & $\begin{array}{c}\text { Engineering } \\
\text { Samples }\end{array}$ & $\begin{array}{c}450 \mathrm{~V} \text { n-type } \\
\text { power } \\
\text { MOSFET } \\
\end{array}$ & VDMOS & $\begin{array}{l}\text { H: (TAMU10DEC) } \\
\text { JML }\end{array}$ & $\begin{array}{l}\text { H: Ag (LET 42) pass/fail } \\
150 \mathrm{~V} / 170 \mathrm{~V} \text {. } \\
\end{array}$ & $\mathrm{OV}_{0 \mathrm{~s}}$ & 3 & [47] \\
\hline 2N7270 & Semicoa & $\begin{array}{c}\text { Engineering } \\
\text { Samples }\end{array}$ & $\begin{array}{c}500 \text { V n-type } \\
\text { power } \\
\text { MOSFET }\end{array}$ & VDMOS & $\begin{array}{l}\text { H: (TAMU10AUG) } \\
\text { JML }\end{array}$ & $\begin{array}{l}\text { H: Tests conducted on multiple } \\
\text { process variations in support } \\
\text { of product development. }\end{array}$ & $\mathrm{OV}_{0 s}$ & 13 & [48] \\
\hline $\begin{array}{l}\text { STRH100N10F } \\
\text { SY3 }\end{array}$ & STMicro & $\begin{array}{l}\text { Engineering } \\
\text { Samples }\end{array}$ & $\begin{array}{c}100 \mathrm{~V} \text { n-type } \\
\text { power } \\
\text { MOSFET }\end{array}$ & VDMOS & $\begin{array}{l}\text { H: (TAMU10AUG, } \\
\text { TAMU10DEC) JML }\end{array}$ & 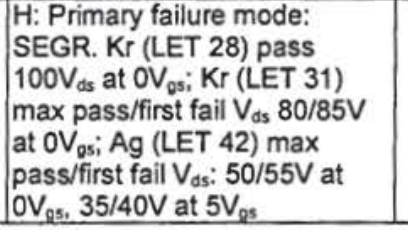 & $0,5 \mathrm{~V}_{0 s}$ & 18 & [49] \\
\hline
\end{tabular}




\begin{tabular}{|c|c|c|c|c|c|c|c|c|c|}
\hline Part Number & Manufacturer & LDC & $\begin{array}{l}\text { Device } \\
\text { Function }\end{array}$ & $\begin{array}{l}\text { Tech- } \\
\text { nology }\end{array}$ & $\begin{array}{c}\text { Particle: } \\
\text { (Facility/Date) P.I. }\end{array}$ & $\begin{array}{l}\text { Test Results LET in } \\
M e V \cdot \mathrm{cm}^{2} / \mathrm{mg} \sigma \text { in } \\
\mathrm{cm}^{2} / \text { device, unless } \\
\text { otherwise specified }\end{array}$ & 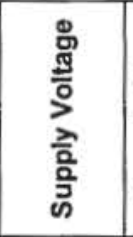 & 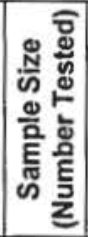 & 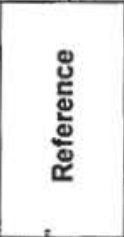 \\
\hline \multicolumn{10}{|l|}{ Miscellaneous: } \\
\hline STAR-250NDR & Cypress & $\begin{array}{c}\text { Custom } \\
\text { test } \\
\text { vehicle }\end{array}$ & $\begin{array}{l}\text { Active Pixel } \\
\text { Sensor }\end{array}$ & CMOS & $\mathrm{H}:$ (TAMU10AUG) JP & $\mathrm{H}: \mathrm{SEL}$ LET $\mathrm{th}_{\mathrm{h}}>77 @ 65^{\circ} \mathrm{C}$ & \begin{tabular}{|c|}
$3.5,5.0$ \\
5.5, and \\
$6.0 \mathrm{~V}$
\end{tabular} & 2 & [50]. \\
\hline PCM A33 & Numonyx & 0930 & $\begin{array}{c}\text { Nonvolatile } \\
\text { Memory }\end{array}$ & $\begin{array}{c}90 \mathrm{~nm} \\
\text { chalcoge } \\
\text { nide } \\
\text { PCM } \\
\end{array}$ & $\begin{array}{l}\text { H: (TAMU1OMAY) } \\
\text { HKITO }\end{array}$ & $\begin{array}{l}\text { H: SEL LET } \text { th }<31.9 ; \\
\text { SEL } \sigma>2.6 \times 10^{-5} \mathrm{~cm}^{2} ; \\
\text { PCM's SELs were from control } \\
\text { circuitries. }\end{array}$ & $3 \mathrm{~V}$ & 8 & [51] \\
\hline $\begin{array}{l}\text { NAND04GW3B } \\
\text { 2DN6 }\end{array}$ & Numonyx & 0831 & $\begin{array}{l}\text { 4G NAND } \\
\text { Flash } \\
\text { Memory }\end{array}$ & $\begin{array}{l}73 \mathrm{~nm} \\
\text { CMOS }\end{array}$ & H: (TAMU10MAY) TO & $\begin{array}{l}\text { H: SEL LET } T_{\text {th }}>56 \text {; SEFIs } \\
\text { observed (control logic errors } \\
\text { on nearly every dynamic mode } \\
\text { shot); } \\
\text { Bit error } \sigma<1 \times 10^{-10} \mathrm{~cm}^{2} / \text { bit. }\end{array}$ & $3.3 \mathrm{~V}$ & 2 & [52] \\
\hline K9F8G08U0M & Samsung & 0807 & \begin{tabular}{|c|} 
8Gbit NAND \\
Flash. \\
Memory \\
\end{tabular} & $\begin{array}{l}60 \mathrm{~nm} \\
\text { CMOS }\end{array}$ & H: (TAMU10DEC) TO & $\begin{array}{l}\text { H: Current Spike Experiment } \\
\text { at high LETs }\end{array}$ & $3.3 \mathrm{~V}$ & 4 & {$[53][54]$} \\
\hline RAM-3+ & Mini-Circuits & 0918 & \begin{tabular}{|c|} 
MMIC \\
Amplifier \\
\end{tabular} & InGaP & L: (NRL10FEB) JP & $\begin{array}{l}\text { L: Sensitive to SETs. No } \\
\text { destructive effects observed. }\end{array}$ & $5 \mathrm{~V}$ & 2 & [55] \\
\hline RAM-6+ & Mini-Circuits & 0927 & $\begin{array}{c}\text { MMIC } \\
\text { Amplifier }\end{array}$ & $\begin{array}{l}\text { Silicon } \\
\text { Bipolar }\end{array}$ & L: (NRL10FEB) JP & $\begin{array}{l}\text { L: Sensitive to SETs. No } \\
\text { destructive effects observed. }\end{array}$ & $3.5 \mathrm{~V}$ & 2 & [56] \\
\hline $\begin{array}{l}\text { HMC422 } \\
\text { (Generic PN); } \\
\text { HMC4562G8 } \\
\text { (Engineering } \\
\text { PN); } \\
\text { HMC4563G8 } \\
\text { (Flight PN) }\end{array}$ & $\begin{array}{l}\text { Hittite } \\
\text { Microwave } \\
\text { Corp. }\end{array}$ & $\begin{array}{l}\text { JX22 } \\
(24 \text { th } \\
\text { week of } \\
2009)\end{array}$ & $\begin{array}{l}\text { GaAs MMIC } \\
\text { mixer with } \\
\text { integrated } \\
\text { LO amplifier }\end{array}$ & MESFET & L: (NRL10JAN) JP & $\begin{array}{l}\text { L: The } 590 \mathrm{~nm} \text { laser pulse } \\
\text { produced SETs under all } \\
\text { conditions tested for both DC } \\
\text { and down conversion from } \\
1530 \mathrm{MHz} \text { to } 30 \mathrm{MHz} \text {. } \\
\text { Transients }<0.5 \mathrm{~V} \text { for } \mathrm{DC} \text { and } \\
\mathrm{AC} \text { input conditions on } \mathrm{RF} \text { and } \\
\mathrm{LO} \text {, with pulse width } 1 \mathrm{~ns} \text { at } \\
10 \% \text { of the peak voltage. }\end{array}$ & $3 \mathrm{~V}$ & 2 & [57] \\
\hline $\begin{array}{c}\text { MT29F4G08AA } \\
\text { AWP }\end{array}$ & Micron & 0748 & $\begin{array}{l}\text { 4Gbit NAND } \\
\text { Flash } \\
\text { Memory }\end{array}$ & $\begin{array}{l}73 \mathrm{~nm} \\
\text { CMOS }\end{array}$ & $\begin{array}{l}\text { H: (TAMU1ODEC) } \\
\text { TO; } \\
\text { L: (NRL11MAR) TO }\end{array}$ & $\begin{array}{l}\text { H: Current Spike Experiment } \\
\text { at high LETs; } \\
\text { L: (NRL11MAR) Sensitive } \\
\text { areas were identified. }\end{array}$ & $3.3 \mathrm{~V}$ & 5 & $\begin{array}{c}{[53][54]} \\
{[58]}\end{array}$ \\
\hline CMOS Test Veh & Intersil & 002 & $\begin{array}{c}\text { Power On } \\
\text { Reset Circuit }\end{array}$ & CMOS & $\begin{array}{l}\text { H: (TAMU1OMAY) } \\
\text { MiC; } \\
\text { L: (NRL10MAY) MiC }\end{array}$ & $\begin{array}{l}\text { H: } 2.8<\text { SET LET } \text { th }<8.6 \text {; } \\
\text { L: SET triggers Reset. }\end{array}$ & $15 \mathrm{~V}$ & 2 & [59] \\
\hline \multicolumn{10}{|c|}{ CMOS Test Vehicle: } \\
\hline Test Vehicle & IBM & $\begin{array}{l}\text { Custom } \\
\text { test } \\
\text { vehicle }\end{array}$ & Latches & $\begin{array}{l}45 \mathrm{~nm} \\
\mathrm{SOI} \\
\mathrm{CMOS}\end{array}$ & $\begin{array}{l}\text { H: (TAMU10MAR) } \\
\text { (LBNL10JUN) JP; } \\
\text { P: (IU10MAR) } \\
\text { (UDC10JUN) JP; Part } \\
\text { was tested throughout } \\
2010 / 2011 \text { at the IBM } \\
\text { VdG facility." }\end{array}$ & $\begin{array}{l}\text { H: SEU LET } \\
\text { destructive effects observed. } \\
\text { dest } \\
\text { TAMU, LBNL, and IBM VdG* } \\
\text { results agree. } \\
\text { P: SEU LET }<1 \text {; Sensitive to } \\
\text { low-energy protons. }\end{array}$ & $0.6-1.0 \mathrm{~V}$ & 3 & [60] \\
\hline Test Vehicle & IBM & $\begin{array}{l}\text { Custom } \\
\text { test } \\
\text { vehicle }\end{array}$ & Latches & $\begin{array}{l}32 \mathrm{~nm} \\
\text { SOl } \\
\text { CMOS }\end{array}$ & $\begin{array}{l}\text { H: (LBNL11JAN) JP; } \\
\text { P: (UDC10OCT) JP; } \\
\text { Part was tested } \\
\text { throughout 2010/2011 } \\
\text { at the IBM VdG } \\
\text { facility. }\end{array}$ & $\begin{array}{l}\text { H: SEU LET } \text { th }_{\text {th }}<2 \text {. No } \\
\text { destructive effects observed. } \\
\text { LBNL and IBM VdG results } \\
\text { agree.* } \\
\text { P: SEU LET } \text { Lth }_{\text {t }}<2 \text {; Sensitive to } \\
\text { low-energy protons. }\end{array}$ & $0.7-1.0 \mathrm{~V}$ & 2 & {$[60]$} \\
\hline Test Vehicle & Intel & $\begin{array}{l}\text { Custom } \\
\text { test } \\
\text { vehicle }\end{array}$ & Latches & $\begin{array}{l}45 \mathrm{~nm} \\
\text { CMOS }\end{array}$ & P: (UDC100CT) JP & $\begin{array}{l}\text { P: Sensitive to low-energy } \\
\text { protons. Irradiations conducted } \\
\text { below nominal operating } \\
\text { voltage. }\end{array}$ & $0.5-0.7 \mathrm{~V}$ & 5 & [61] \\
\hline Test Vehicle & Intel & $\begin{array}{l}\text { Custom } \\
\text { test } \\
\text { vehicle }\end{array}$ & Latches & $\begin{array}{l}32 \mathrm{~nm} \\
\text { CMOS }\end{array}$ & P: (UDC100CT) JP & $\begin{array}{l}\text { P: Sensitive to low-energy } \\
\text { protons. Irradiations conducted } \\
\text { below nominal operating } \\
\text { voltage. }\end{array}$ & $0.7 \mathrm{~V}$ & 5 & [61] \\
\hline
\end{tabular}

- The IBM T.J. Watson Research Center has a 3 MV Tandem Van de Graaff accelerator that is used for SEU experiments. The lab has seven beam lines, one of which is dedicated to SEU exposures. The ion sources for the accelerator consist of a sputter source (SNICS) for $1 \mathrm{H}, 2 \mathrm{H}, 12 \mathrm{C}$ and other beams as well as a RF source (alphatross) used to produce alpha particles (4He). [IBM VdG-Heidel] 


\section{TEST RESULTS AND DISCUSSION}

As in our past workshop compendia of NASA Goddard Space Flight Center (GSFC) test results, each DUT has a detailed test report available online at http://radhome.gsfc. nasa.gov [11] describing the test method, SEE conditions/ parameters, test results, and graphs of data.

This section contains summaries of testing performed on a selection of featured parts.

\section{A. Vishay SiB455EDK and Si7431DP Commercial Power MOSFETS}

This study was undertaken to determine the SEGR and SEB susceptibility of Vishay's commercial p-type trenchFET® power MOSFETs under heavy-ion irradiation. The SiB455EDK is a $12 \mathrm{~V}, 9 \mathrm{~A}$ p-channel trench MOSFET from Vishay's Gen III line. The Si7431DP is a $200 \mathrm{~V}, 2.2 \mathrm{~A} \mathrm{p}$ channel trench MOSFET introduced in 2005. Past reports of trench power MOSFETs have focused on n-channel devices and the substantial degradation from localized dose deposited by heavy ions [63] [64]. We believe the test reported here is the first to evaluate the suitability of p-channel trench MOSFETs for space applications.

The SiB455EDK tests were conducted in air at three different angles $\left(2^{\circ}, 45^{\circ}\right.$, and $\left.60^{\circ}\right)$; the Si7431DP tests were conducted in vacuum at normal beam incidence, with additional tests at $45^{\circ}$ and $60^{\circ}$ under $1230 \mathrm{MeV} \mathrm{Kr}$ only. Each sample was biased at one of three gate-source voltage biases, and the drain-source voltage was incremented between beam runs until device failure was observed. Currents were monitored at the gate and drain nodes during testing, and a post-irradiation gate stress test was performed following each beam run. Failure was defined as the gate current exceeding the vendor specified maximum leakage current, and/or a sudden, sustained increase in drain current. In addition, the gate threshold voltage was measured following each beam run to monitor for dose effects. No dosing effects were seen during heavy-ion testing, and as reported in [2], the SiB455EDK showed a TID tolerance to more than 100 krad(Si) with $\mathrm{C} 060$ irradiation.

The primary failure mode of the SiB455EDK was SEGR, although in some samples irradiated at $2^{\circ}$ a SEB signature was detected that also resulted in gate damage. The SEE response curve for the $2^{\circ}$ angle of incidence is shown in Fig. 1; the data obtained at higher angles are plotted in Fig. 2.

The primary failure mode of the Si7431DP was SEGR. The SEE response curve for data taken at normal incidence is shown in Fig. 3. In this figure, the krypton data point at $10 \mathrm{~V}_{\mathrm{gs}}$ reflects the lowest failure $\mathrm{V}_{\mathrm{ds}}$ : two samples passed at $-120 \mathrm{~V}_{\mathrm{ds}}$, failing at the next bias step, but the third sample failed during the first beam run at $-110 \mathrm{~V}_{\mathrm{ds}}$. At all three $\mathrm{V}_{\mathrm{gs}}$ biases, no failures were detected at $45^{\circ}$ and $60^{\circ}$ angles of $1230 \mathrm{MeV}$ krypton beam incidence.

The results suggest that these Vishay commercial p-type trench power MOSFETs are a consideration for space applications. [45] [46]

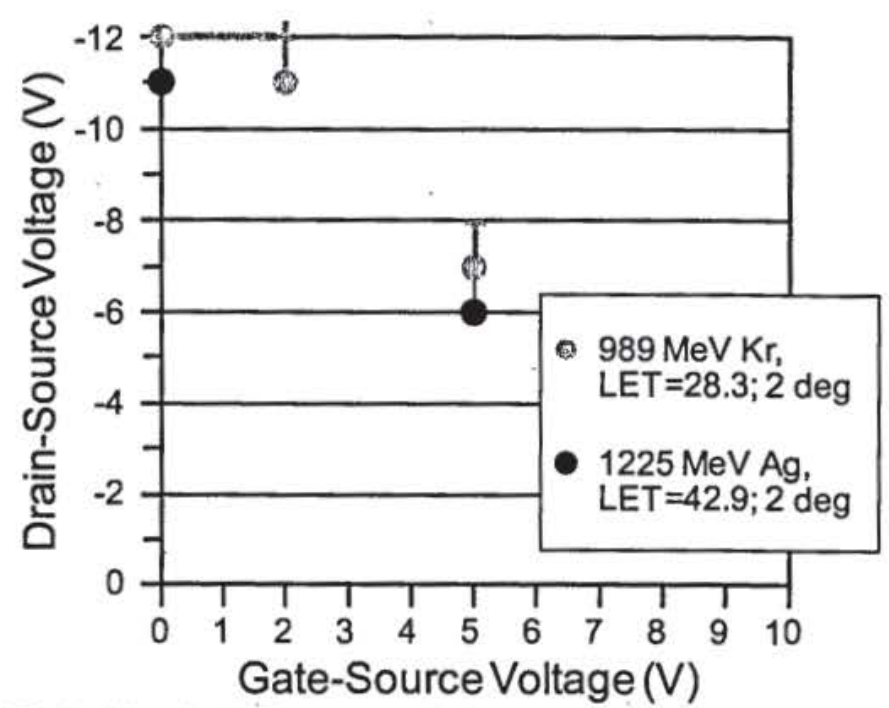

Fig. 1. Vishay SiB455EDK Commercial Power MOSFET SEE response curve for the $2^{\circ}$ angle of incidence.

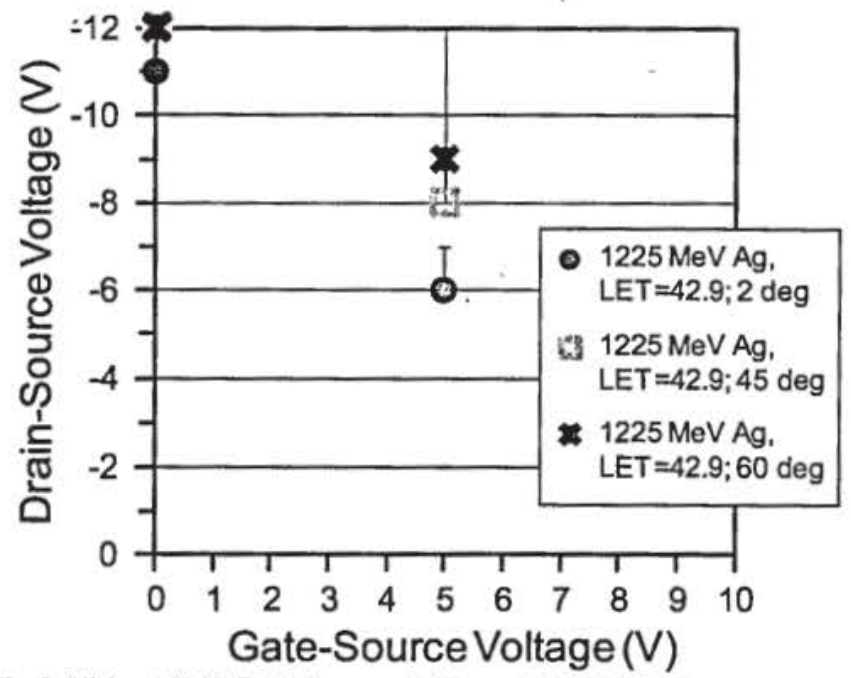

Fig. 2. Vishay SiB455EDK Commercial Power MOSFET SEE response curve for the higher angles incidence.

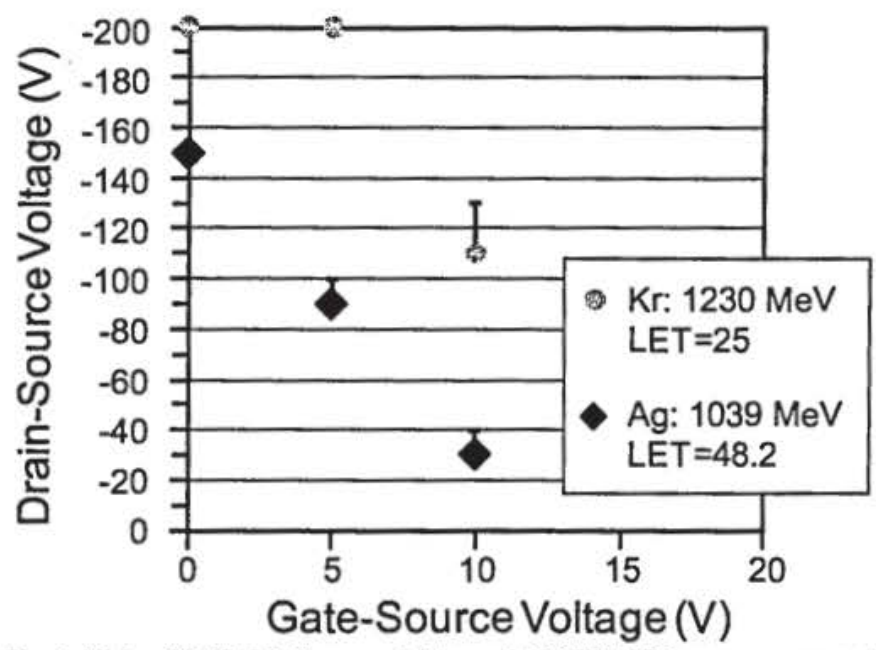

Fig. 3. Vishay Si7431DP Commercial Power MOSFET SEE response curve at normal incidence. 


\section{B. PE9915X Monolithic Point-of-Load Switching Regulator}

The PE9915X is a series of monolithic point-of-load (POL) switching regulators fabricated in Peregrine's UltraCMOS® technology, which utilizes a Silicon-On-Sapphire (SOS) process. We examined the PE99151 (2 A) and the PE99155 $(10 \mathrm{~A})$ versions. The output voltage is adjustable down to $1 \mathrm{~V}$. The manufacturer supplied customized evaluation boards for the test, which consists of external resistors, capacitors, and an inductor. We note that the device has a $1000 \mu \mathrm{F}$ capacitor at the output.

Three parts were tested at the LBNL facility. The test was performed in a vacuum environment. The application boards were attached to metal plates to facility conductive heat sinking. Fig. 4 shows a block diagram of the test setup and Fig. 5 shows the photograph of the device inside the test chamber.

We irradiated the parts with $10 \mathrm{MeV} / \mathrm{amu} \mathrm{Xe}$ at $0^{\circ}$ and $60^{\circ}$ for different output load conditions. We observed a few incidences of sharp voltage spikes, with magnitudes of 0.4 to $0.6 \mathrm{~V}$ and durations on the order of $10 \mathrm{~s}$ of nanoseconds. However we believe that these signatures originated from sources external to the device and test board. The resonating frequency of the pulse and the short duration suggest that the voltage spikes were likely caused by noise associated with the vacuum chamber and/or equipment in the irradiation room.

We also observed an incidence where the input supply current fell rapidly, which caused an output regulation failure, as shown in Fig. 6. However we believe that the event was not caused by a single particle strike. It may have been caused in part by device overheating, as conductive heat-sinking becomes inefficient in a vacuum environment. The parametric degradations from accumulated dose may have also contributed to the current drop. We observed parametric shifts during electrical characterization approximately 48 hours after the heavy-ion test. Additional tests in air, eliminating the heat sinking issues were performed to verify the cause of the event. [17]

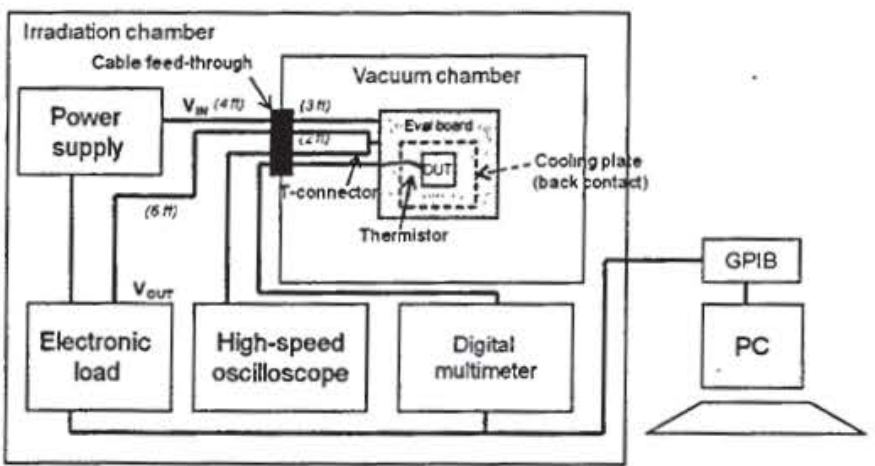

Fig. 4. Block diagram of the testing setup for the PE9915X Monolithic Pointof-Load Switching Regulator.

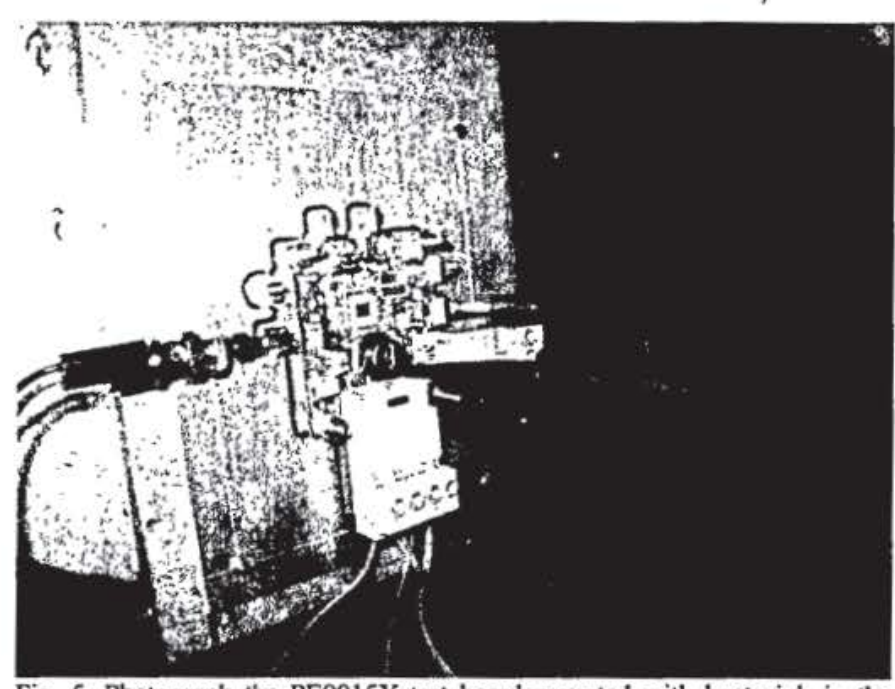

Fig. 5. Photograph the PE9915X test board mounted with heat sink in the irradiation chamber.

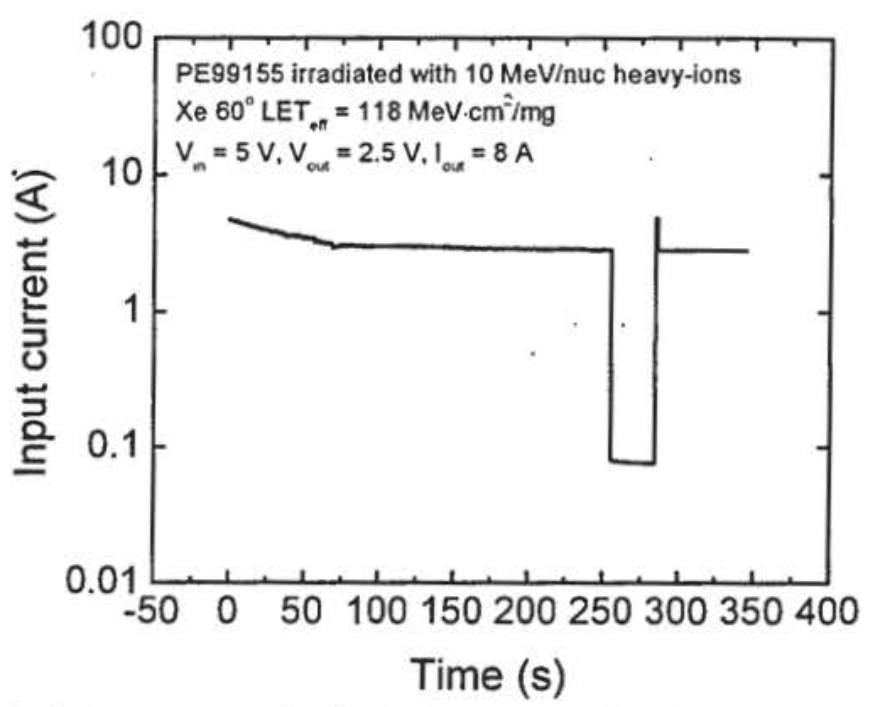

Fig. 6. Input current vs. time for the PE99155, operating with Vout $=2.5 \mathrm{~V}$ and $\mathrm{I}_{\text {out }}=8 \mathrm{~A}$, irradiated with $\mathrm{Xe}$ at $60^{\circ}$ with an effective $\mathrm{LET}=118$ $\mathrm{MeV} \cdot \mathrm{cm}^{2} / \mathrm{mg}$, for $10 \mathrm{MeV} / \mathrm{nuc}$ heavy-ions.

\section{Hittite HMC422 Double-Balanced Mixer Integrated Circuit}

The Hittite HMC422 is a double-balanced mixer integrated circuit with integrated local oscillator (LO) amplifiers fabricated as a monolithic microwave integrated circuit in a gallium arsenide $(\mathrm{GaAs})$ process. This mixer can operate as an upconverter or downconverter between $1.2 \mathrm{GHz}$ and $2.5 \mathrm{GHz}$. With the integrated LO amplifier, the mixer requires an LO drive level of only $0 \mathrm{dBm}$, and requires only $30 \mathrm{~mA}$ from a single positive $3 \mathrm{~V}$ rail. The mixer has $8 \mathrm{~dB}$ of conversion loss, an input $\mathrm{P} 1 \mathrm{~dB}$ of $+8 \mathrm{dBm}$ and an input third order intercept point of $+15 \mathrm{dBm}$ at $2 \mathrm{GHz}$. The device was monitored for analog single-event transients (ASETs) on the IF output and for any destructive events induced by exposing it to a pulsed laser beam at the NRL laser test facility. The application being tested was used to downconvert a $1530 \mathrm{MHz}$ $\mathrm{CW}$ signal to $30 \mathrm{MHz}$ continuous wave $(\mathrm{CW})$ by supplying inputs to RF and LO while monitoring IF with the oscilloscope. 
The device was prepared for test by depotting the topside of the plastic package via acid etching and then mounting it onto a Hittite evaluation board (part number 104813) that uses a Rogers 4350 substrate and $50 \Omega$ signal line impedance. A finished 1 inch by 1 inch square test board is shown in Fig. 7.

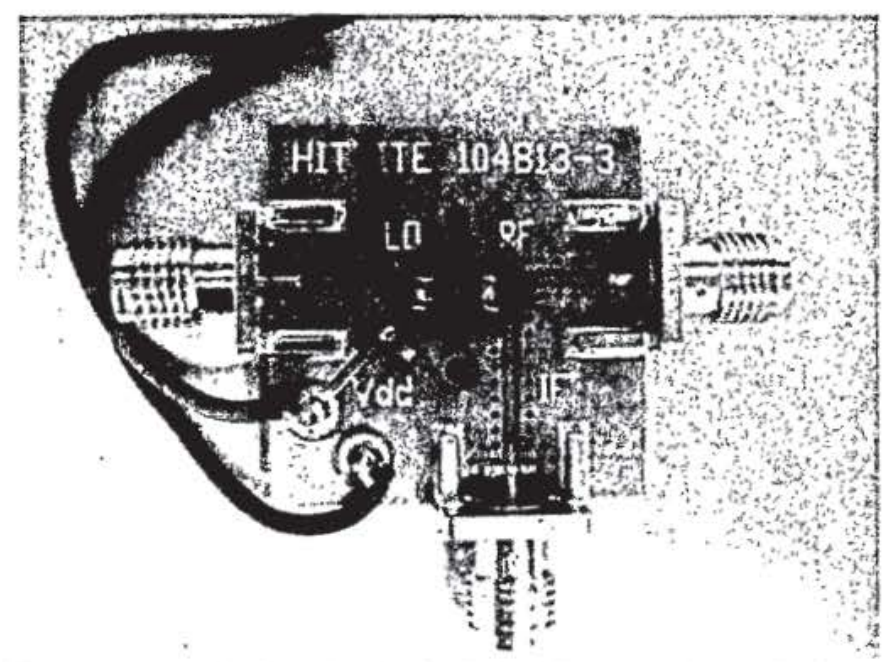

Fig. 7: Photograph of completed printed circuit board ready for pulsed laser testing. The device under test is in the center of the board and the surfacemount connectors on the three edges are SMA type. The $\mathrm{V}_{d d}$ power supply ( 3 $\mathrm{V}$ ) as well as the LO, IF, and RF signal lines are marked.

The tests were performed on two devices at the NRL using their single-photon absorption (SPA) setup. This system has been discussed in several refereed publications and will not be covered in detail [65] [66] [67]. The device lot date code was JX22. The DUT was packaged in a plastic MSOP-8 package with a copper alloy lead frame. The test setup consisted of laptop running LabVIEW for instrument control and data capture, a Tektronix DSA72004B digital storage oscilloscope (20 GHz, $50 \mathrm{GS} / \mathrm{s})$, two HP 83712B synthesized CW generators, and an Agilent N6702A MPS mainframe 4channel power supply. A separate function generator specific to the NRL test facility was used to synchronize the two GSFC function generators to the delivery of the laser pulse so that different points within the clock period could be sampled. The two function generators had their $10 \mathrm{MHz}$ synchronization lines tied to the NRL function generator sync $1 / O$. This allowed the laser pulse firing to be used as the trigger source for the oscilloscope enabling the laser pulse to move throughout all $2 \pi$ of the CW input on RF and LO.

The laser was focused onto the HMC422 die and scanned across the surface to produce transients on the IF output. This was done for two different input (RF and LO) conditions: 1) biased operation with the RF and LO inputs terminated, and 2) biased operation with RF and LO inputs stimulated with 1530 $\mathrm{MHz}$ and $1500 \mathrm{MHz} \mathrm{CW}$ sine wave signals at $0 \mathrm{dBm}$. This was not an application specific test, but the input frequencies were relevant for the end users. The parameters varied were the RF and LO input conditions and the laser pulse energy. Transients were captured for all test conditions and are shown in Fig. 8.

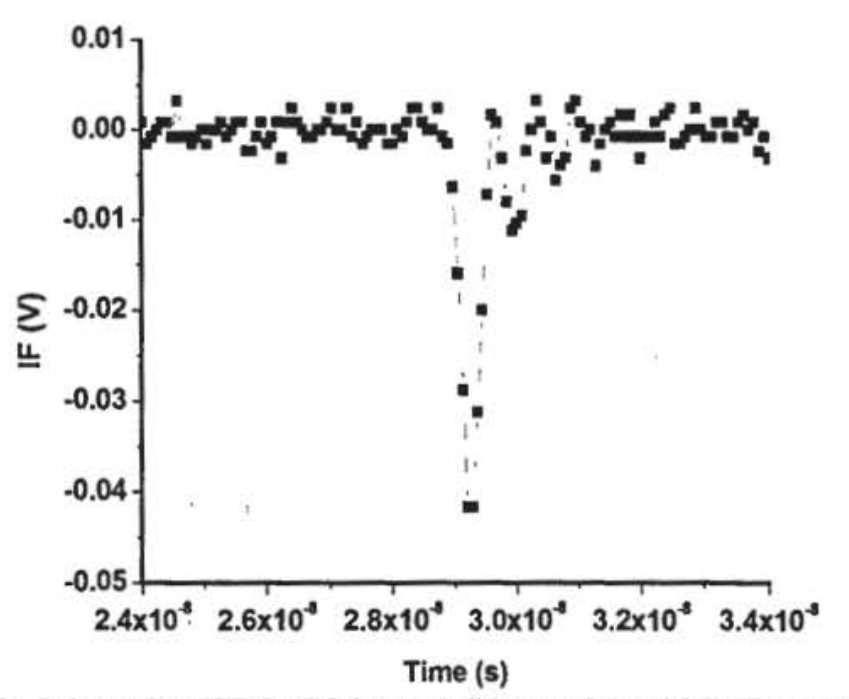

Fig. 8: Example ASET for DC input conditions on RF and LO. The GaAs MESFETs in the center of the die were the only source of transients - the LO amplifier did not appear to contribute. All transients are negative. These transients were recorded at a laser pulse energy of approximately $33 \mathrm{pJ}$.

The Hittite HMC422 tested here showed transients less than $0.5 \mathrm{~V}$ in amplitude for both $\mathrm{DC}$ and $\mathrm{AC}$ input conditions on $\mathrm{RF}$ and $\mathrm{LO}$ up to a laser pulse energy of $33 \mathrm{pJ}$. The transients widths, measured at $10 \%$ of the peak voltage, were approximately $1 \mathrm{~ns}$. The DC input condition ASETs measured on IF appeared to be simply superimposed on the IF signal and caused no further distortion during AC input conditions. The amplitude and width remained unchanged as shown in Fig. 9.

GaAs technologies generally suffer from large single-event cross sections due to large charge collection efficiencies and high carrier mobility. These tests seem to indicate that the response saturated quickly with increasing laser pulse energy.[57]

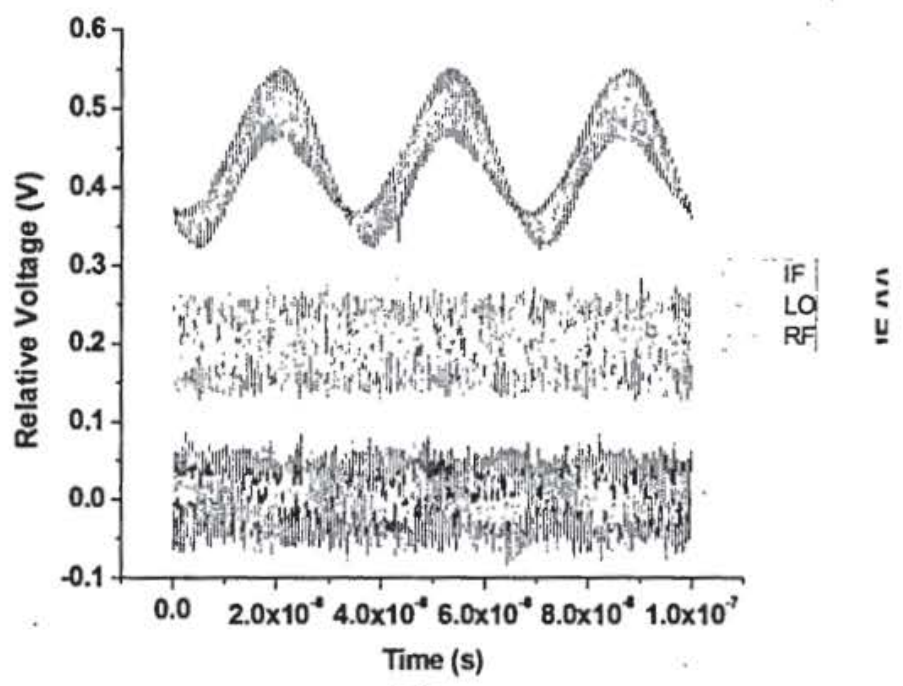

(a) 


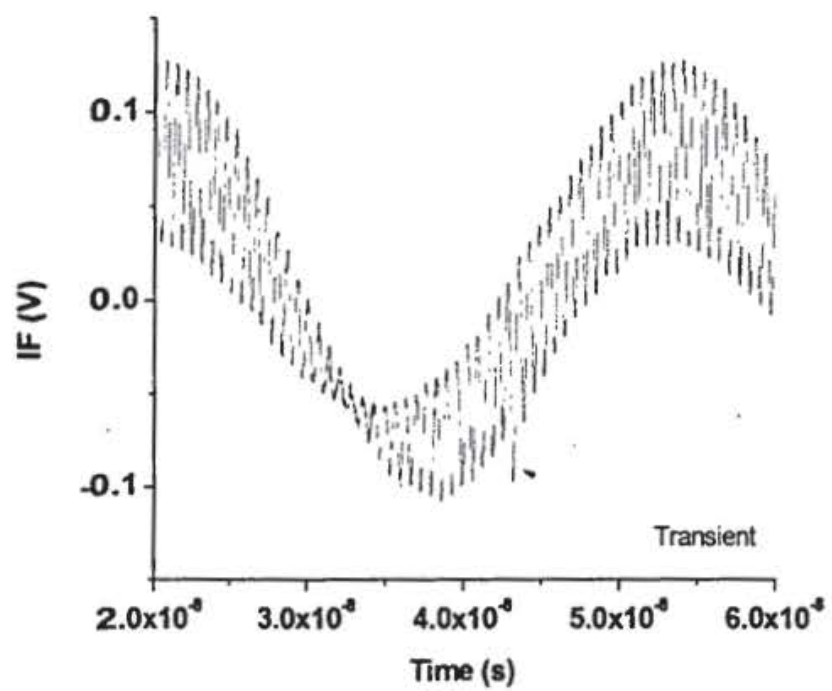

(b)

Fig. 9: These figures show ASETs for AC RF and LO input characteristics at a pulse energy of $33 \mathrm{pJ}$. (a) shows an ASET on IF as well as the raw RF and LO signals from the CW generators; (b) shows a zoomed image of the ASET from the top trace of (a). Note that the voltages shown in (a) are arbitrary and simply relative within each trace's range - the data were stacked to show them on the same abscissa.

\section{MSK5978RH LDO Positive Adjustable Regulator}

This study was undertaken to determine the ASET susceptibility of the M. S. Kennedy MSK5978RH low dropout (LDO), positive, $0.7 \mathrm{~A}$, adjustable linear regulator. The device was monitored for transients on the voltage output while exposing it to a pulsed laser beam at the NRL laser test facility,

The sensitive structure within the MSK5978RH is the Linear Technology RH3080, which integrates a PNP pass transistor and the regulation control loop circuitry onto a single die; this makes this part fundamentally different from previous $\mathrm{RH} 1573 \mathrm{~K} /$ pass transistor combinations in other LDO regulator offerings from M.S. Kennedy and Linear Technology. A photograph of the die is shown in Fig. 10.

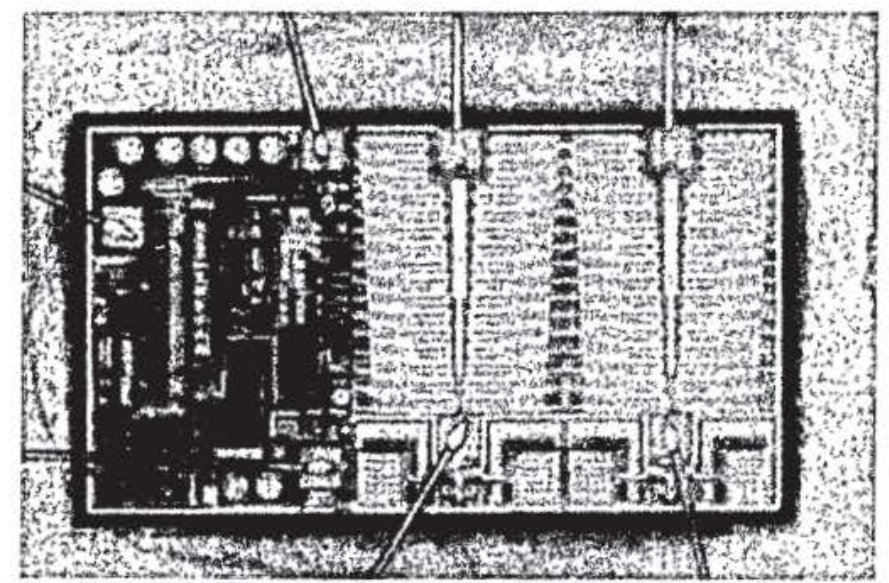

Fig. 10: MSK5978RH photomicrograph of the RH3080 die inside the hybrid package. The pass transistor is on the left, while the controller circuitry (current source, voltage follower, etc.) is on the left. The pass transistor is not sensitive to SETs, but the controller circuitry can produce ASETs. This was the primary target of the pulsed laser. wavelength laser pulse with a pulse width of approximately 1 ps. Both $100 \mathrm{x}$ and $10 \mathrm{x}$ objectives were used to focus the beam onto the surface of the die yielding spot sizes between 1.2 and $12 \mu \mathrm{m}$ in diameter. The difference in spot size did not affect the transient behavior. The pulse energy was maintained at 4.6 $\mathrm{pJ}$, which is sufficiently conservative for a worst-case analysis.

The laser spot was scanned across the controller circuitry and the ASETs were captured and recorded using a $500 \mathrm{MHz}$ oscilloscope (Tektronix MSO4054B). The application conditions required that we test the LDO regulator under several input bias and output load conditions. Fig. 11 shows the characteristic amplitude and width for ASETs under two different conditions: 1) $16 \mathrm{~V}$ input, $15 \mathrm{~V}$ output, and $150 \mathrm{~mA}$ load; and, 2) $2.2 \mathrm{~V}$ input, $1.5 \mathrm{~V}$ output, and $180 / 500 \mathrm{~mA}$ loads. In addition to 0.1 and $1.0 \mu \mathrm{F}$ ceramic surface-mount capacitors, the output was loaded with a $22 \mu \mathrm{F}$ tantalum capacitor (Kemet T495D226K035ATE250) with an equivalent series resistance of approximately $0.25 \Omega$.

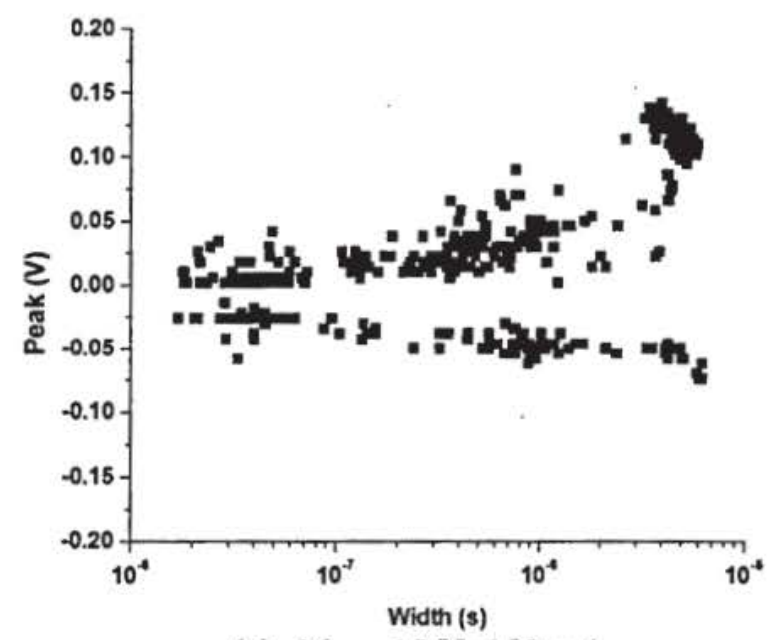

(a) $16 \rightarrow 15 \mathrm{~V} ; 150 \mathrm{~mA}$

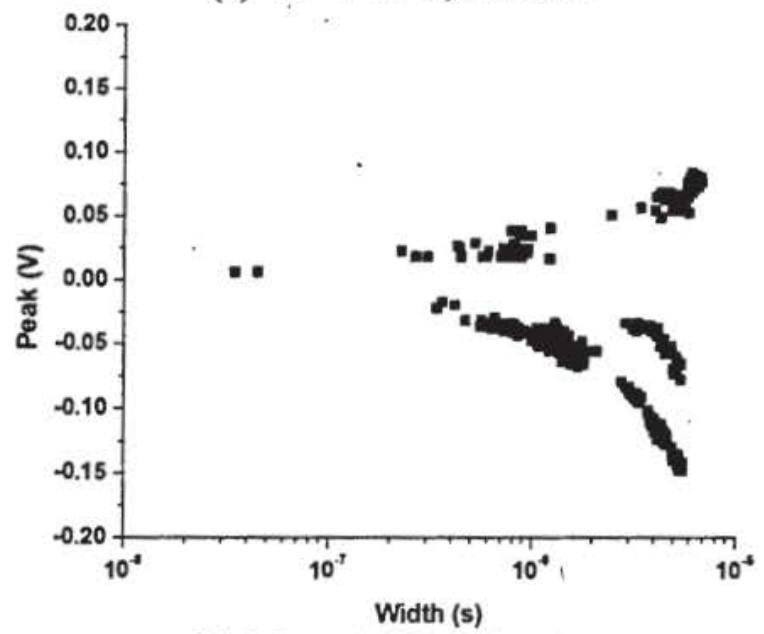

(b) $2.2 \rightarrow 1.5 \mathrm{~V} ; 500 \mathrm{~mA}$

Fig. 11: ASET transient characteristics for two different input and output conditions. The voltage on the ordinate is the peak amplitude of the ASET, which is the larger of the two excursions from $0.0 \mathrm{~V}$ if the ASET is bipolar. The width is evaluated at $10 \%$ of the peak voltage. 
As with other M. S. Kennedy LDO regulators, this one is sensitive to ASETs and may require filtering techniques to reduce the transient amplitude at the expense of increasing the transient width. The ASETs reported here are applicationspecific and are also a function of the radiation source used; a different setup and a heavy ion source would yield different ASET behavior. However, given its smaller size due to increased integration and lower heat dissipation, it may be an attractive option for certain applications. [31]

\section{E. MT29F4G08AAAWP Micron 4Gbit NAND}

In an effort to determine which regions on the die cause the high currents observed when testing at the TAMU Cyclotron, we conducted tests using the NRL pulsed laser on the MT29F4G08AAAWP Micron 4Gbit NAND. We used single photon absorption, shining $590 \mathrm{~nm}$ (green) light on the front surface of the Micron 4G NAND flash memory. In Fig. 12, we show the results of the pulsed laser test at NRL. Light spots indicate locations where high current, $80 \mathrm{~mA}$ or more, was observed. Dark spots indicate locations where SEFIs, without high current, were observed. There are 38 locations where high currents were triggered by the laser, including almost anywhere in the peripheral control logic. Note, however, that there are no high current events in the regions believed to be the charge pumps (white rectangles). This result is very difficult to reconcile with the conclusion in $[68,69]$, that the high currents are coming from the charge pumps. [53, 54, 58]

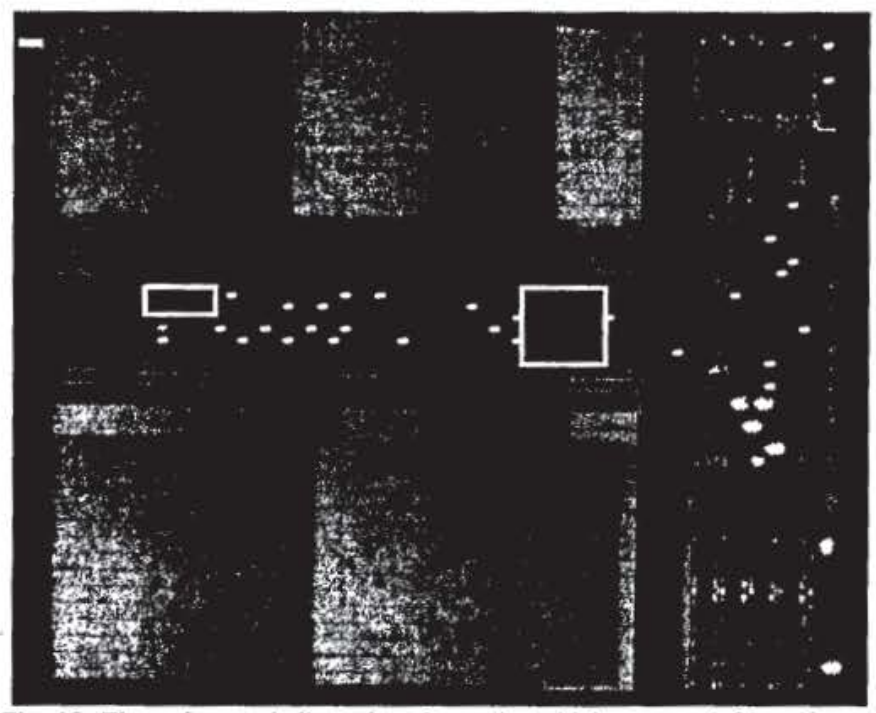

Fig. 12. The red spots indicate locations where high current $(>80 \mathrm{~mA})$ was observed. Other colors indicate different kinds of SEFIs, without high current.

\section{SUMMARY}

We have presented current data from SEE testing on a variety of mainly commercial devices. It is the authors' recommendation that this data be used with caution. We also highly recommend that lot testing be performed on any suspect or commercial device.

\section{ACKNOWLEDGMENT}

The authors gratefully thank members of the Radiation Effects and Analysis Group who contributed to the test results presented here: Mark R. Friendlich, Donald K. Hawkins, Alvin J. Boutte, Edward P. Wilcox, James D. Forney, Timothy L. Irwin, Donna J. Cochran, Christina M. Seidleck, Megan C Casey, Stephen R. Cox, Christopher Perez, Martin A. Carts, and Joseph W. Portner. The authors also thank Dale McMorrow and Stephen P. Buchner for their expert help with laser testing at NRL. Special thanks to Rafi Albarian and Sam Burns from Linear Technology, as well as Greg Overend and Paul Musil from M. S. Kennedy Corporation for their help and generosity in providing test hardware. Also, special thanks to Géraldine Chaumont, $\mathrm{Al}$ Ouellet, and Patrick Briand from STMicro for their generous provision of test vehicles; and Dave MacDonald and John Demiray from Vishay Intertechnology for their support of testing the trench-type MOSFETs.

\section{REFERENCES}

[1] Kenneth A. LaBel, Lewis M. Cohn, and Ray Ladbury,"Are Current SEE Test Procedures Adequate for Modern Devices and Electronics Technologies?," http://radhome.gsfc.nasa.gov/ radhome/ papers/ HEART08_LaBel.pdf

[2] Donna J. Cochran, Dakai Chen, Timothy Oldham, Hak Kim, Stephen Buchner, Kenneth A. LaBel, Jonathan Pellish, Martin A. Carts, Martha V. O'Bryan, and Anthony B. Sanders, " Recent Total lonizing Dose and Displacement Damage Compendium of Candidate Electronics for NASA Space Systems," submitted for publication in IEEE Radiation Effects Data Workshop, Jul. 2011.

[3] Lawrence Berkeley National Laboratory (LBNL), 88-Inch Cyclotron Accelerator Facility, http://www-afrd.lbl.gov, Feb. 2011.

[4] B. Hyman, "Texas A\&M University Cyclotron Institute, K500 Superconducting Cyclotron Facility," http://cyclotron.tamu.edu' facilities.htm, Jul. 2003.

[5] W.J. Stapor, "Single-Event Effects Qualification," IEEE NSREC95 Short Course, sec. II, pp 1-68, Jul. 1995.

[6] C. M. Castaneda, "Crocker Nuclear Laboratory (CNL) Radiation Effects Measurement and Test Facility," IEEE NSREC01 Data Workshop, pp. 77-81, Jul. 2001.

[7] C. C. Foster, S. L. Casey, P. Miesle, N. Sifri, A. H. Skees, K. M. Murray, "Opportunities for Single Event and Other Radiation Effects Testing and Research at the Indiana University Cyclotron Facility," IEEE NSREC96 Data Workshop, pp. 84-87, Jul. 1996.

[8] J. S. Melinger, S. Buchner, D. McMorrow, T. R. Weatherford, A. B. Campbell, and H. Eisen, "Critical evaluation of the pulsed laser method for single event effects testing and fundamental studies," IEEE Trans. Nucl. Sci., vol 41, pp. 2574-2584, Dec. 1994.

[9] D. McMorrow, J. S. Melinger, and S. Buchner, "Application of a Pulsed Laser for Evaluation and Optimization of SEU-Hard Designs," IEEE Trans. Nucl. Sci., vol 47, no. 3, pp. 559-565, Jun. 2000.

[10] R. Koga and W. A. Kolasinski, "Heavy lon-Induced Single Event Upsets of Microcircuits; A Summary of the Aerospace Corporation Test Data," IEEE Trans. Nucl. Sci., Vol. 31, No. 6, pp. 1190-1195, Dec. 1984.

[11] NASA/GSFC Radiation Effects and Analysis home page, http://radhome.gsfc.nasa.gov

[12] NASA Electronic Parts and Packaging (NEPP) web site, http://nepp.nasa.gov/.

[13] Jonathan Pellish, Raymond Ladbury, Alvin Boutte, "Analog Devices AD7847SQ/883B 12-bit MDAC Single-Event Latchup Characterization Report," http://radhome.gsfc.nasa.gov/ radhome/ papers/ T120510_AD7847SQ.pdf, Dec. 2010.

[14] Jonathan Pellish, "Heavy Ion Single-Event Latchup Characterization of the Texas Instruments ADS7881 Analog-to-Digital Converter," http://radhome.gsfc.nasa.gov/ radhome/ papers/ T110910_ADS7881.pdf, Oct. 2010.

To be presented by Martha O'Bryan at the International Electrical and Electronics Engineering (IEEE) Nuclear and Space Radiation Effects Conference (NSREC), July 27, 2011, Las Vegas, NV, and published on nepp.nasa.gov and radhome.gsfc.nasa.gov. 
[15] Melanie Berg, Hak Kim, Mark Friendlich, Chris Perez, Christina Seidlick, and Ken Label, "LTC1864L Single Event Effects (SEE) Test Report," http://radhome.gsfc.nasa.gov/ radhome/ papers/ T081610_LTC1864L.pdf, Aug. 2010.

[16] Michael Campola, Melanie Berg, Hak Kim, Chris Perez, "Single Event Effects (SEE) Testing of the 7872ARPFS 14-bit Analog to Digital Converter," http://radhome.gsfc.nasa.gov/ radhome/ papers/ T022210_AD7872.pdf, Feb. 2010.

[17] Dakai Chen, Jim Swonger, and Anthony Phan, "Test Report for Heavy Ion Testing of the PE9915X Point-of-Load Switching Regulator," http://radhome.gsfc.nasa.gov/ radhome/ papers/ LBNL012111_ PE9915X.pdf, Jan. 2011.

[18] Dakai Chen, Ted Wilcox, and James Forney, "Test Report for Heavy lon Testing of the MSK5059RH Step Down Switching Regulator," http://radhome.gsfc.nasa.gov/ T120510_MSK5059RH.pdf, Dec. 2010.

[19] Michael Campola, Jonathon Pellish, Anthony Phan, Hak Kim, Tony Sanders, and Mike Xapsos, "Heavy Ion Single Event Transient (SET) Testing of the MSK5820-1.8KRH Radiation Hardened Ultra Low Drop Out Fixed Positive Linear Voltage Regulator," http://radhome. gsfc.nasa.gov/ radhome/ papers/ T052210_MSK5820.pdf, May 2010.

[20] Anthony B. Sanders, Hak S. Kim, and Anthony M. Phan, "Heavy ion SEE test of SMTR28515T from Interpoint,' http://radhome. gsfc.nasa.gov/ radhome/ papers/ LBNL060910_TAMU110910 SMRT28515T.pdf, Jun. Nov. 2010.

[21] Anthony B. Sanders, Hak S. Kim, and Anthony M. Phan, "Heavy ion SEE test of SMTR283R3S from Interpoint," http://radhome. gsfc.nasa.gov/ radhome/ papers/ T030210_SMTR283R3S.pdf, Mar. 2010.

[22] Anthony B. Sanders, Hak S. Kim, and Anthony M. Phan, Heavy ion SEE test of SMTR283R3S from Interpoint," http://radhome. gsfc.nasa.gov/ radhome/ papers/ T030210_ T110910_ SMTR283R3S.pdf, Mar. Nov. 2010.

[23] Anthony B. Sanders, Michael J. Campola, Hak S. Kim, and Anthony M. Phan, "Heavy ion SEE test of SMSA2812D from Interpoint," http://radhome.gsfc.nasa.gov/ radhome/ papers/ T030210 SMSA2812D.pdf, Mar. 2010.

[24] Anthony B. Sanders, Michael J. Campola, Hak S. Kim, and Anthony M. Phan, "Heavy ion SEE test of SMSA2812D from Interpoint," http://radhome.gsfc.nasa gov/ radhome/ papers/ T030210_T110910_ SMSA2812D.pdf, Mar. Nov. 2010.

[25] Anthony B. Sanders, Hak S. Kim, Anthony M. Phan, and Christina Seidleck, "Heavy ion SEE test of MFP0507S from Interpoint," http://radhome.gsfc.nasa.gov/ radhome/ papers/ T120510 LBNL012111_MFP0507S.pdf, Dec. 2010.

[26] Anthony B. Sanders, Dakai Chen, Hak S. Kim, Anthony M. Phan, and Bertrand Nkei, "SEE Transient Response of Crane Interpoint Single Output Point of Load DC-DC Converters," submitted for publication at RADECS 2011, Sept. 2011.

[27] Anthony B. Sanders, Hak S. Kim, and Anthony M. Phan, "Heavy ion SEE test of SLH2812D from Interpoint," http://radhome.gsfc.nasa.gov/ radhome/ papers/ LBNL060910_SLH2812D.pdf, Jun. 2010.

[28] Anthony B. Sanders, Hak S. Kim, and Anthony M. Phan, "Heavy ion SEE test of SLH2812D from Interpoint," http://radhome.gsfc.nasa.gov/ radhome/ papers/ LBNL060910_T110910_SLH2812D.pdf, Jun. Nov. 2010.

[29] Anthony B. Sanders, Hak S. Kim, Anthony M. Phan, "Heavy ion SEE test of SMFLHP2815S from Interpoint," http://radhome.gsfc.nasagov/ radhome/ papers/ T030210_SMFLHP 2815S.pdf, Mar. 2010.

[30] Anthony B. Sanders, Hak S. Kim, and Anthony M. Phan, "Heavy ion SEE test of SMFLHP2815S from Interpoint," http://radhome. gsfc.nasa.gov/ radhome/ papers/ T030210_T110910_ SMFLHP 2815S.pdf, Mar. Nov. 2010.

[31] J. A. Pellish, E. (Ted) P. Wilcox, and S. Buchner, "Pulsed Laser SingleEvent Transient Testing of the M.S. Kennedy MSK5978RH LDO Regulator," http://radhome.gsfc.nasa.gov/ radhome/ papers/ NRL102210 MSK5978.pdf, Nov. 2010.

[32] Anthony B. Sanders, Jonathan Pellish, Hak S. Kim, and Anthony Phan, "Laser Test of the S2803R3S DC/DC Converter," http://radhome. gsfc.nasa.gov/ radhome/ papers/ NRL051110_S2803R3S.pdf, May 2010.

[33] Anthony B. Sanders, Jonathan Pellish, Hak S. Kim, and Anthony Phan, "Laser Test of the SMSA2815S DC/DC Converter," http://radhome. gsfc.nasa.gov/ radhome/ papers/ NRL051110_SMSA2815S.pdf, May 2010.
[34] Melanie Berg, Hak Kim, Mark Friendlich, Chris Perez, Christinia Seidlick, and Ken Label, "Actel ProASIC Field Programmable Gate Array Single Event Effects (SEE) High-Speed Test - Phase I," http://radhome.gsfc.nasa.gov/ radhomel papers/ T052110 A3PE3000.pdf, Mar. 2010.

[35] M. D. Berg, H. Kim, M. Friendlich, C. Perez, C. Seidleck, and K. LaBel, "Incorporating Probability Models of Complex Test Structures to Perform Technology Independent FPGA Single Event Upset Analysis," NSREC PG-1 submitted for publication in IEEE Trans. Nucl. Sci., Dec. 2011.

[36] Melanie Berg, Hak Kim, Mark Friendlich, Chris Perez, Christina Seidlick, and Ken Label, "RTAX2000-S Field Programmable Gate Array Single Event Effects (SEE) High-Speed Test - Phase 1," http://radhome.gsfc.nasa.gov/ radhome/ papers/ T120809_ RTAX2000S.pdf, May Aug. 2010.

[37] M. Berg, H. Kim, C. Perez, M. Friendlich, P. Marshall, D. McMorrow, and K. LaBel, "Achronix SPD60 Heavy Ion Single Event Effects Test Report," http://radhome.gsfc.nasa.gov/ radhome/ papers/ T030210_ SPD60.pdf, Mar. 2010.

[38] Melanie Berg, M. Friendlich, C. Perez, H. Kim, and K. LaBel, "Taming the SEU Beast - Approaches and Results for FPGA Devices and How To Apply Them," NASA Electronic Parts and Packaging (NEPP) Program Electronic Technology Workshop, http://radhome.gsfc. nasa.gov/ radhome/ papers/ NEPP_ETW2011_Berg.pdf, Jun, 2011.

[39] Melanie Berg, and Ken LaBel, "Test and Evaluation of the Radiation Response of Commercial and Hardened Microelectronics - FPGA Test and Evaluation," NASA Electronic Parts and Packaging (NEPP) Program \& Defense Threat Reduction Agency (DTRA) review, http://radhome.gsfc.nasa.gov/ radhome/ papers/ NEPP DTRA_04062010_Berg.pdf, Apr. 2010.

[40] Jonathan Pellish, "Heavy Ion Single-Event Latchup Characterization of the Linear Technology LTC2054HV Single Micropower Zero-Drift Operational Amplifier," http://radhome.gsfc. nasa.gov/ radhome/ papers/ T110910_LTC2054HV.pdf, Nov. 2010.

[41] . Michael Campola, Jonathon Pellish, Anthony Phan, Hak Kim, Tony Sanders, and Mike Xapsos, "Pulsed LaserSingle Event Transient (SET) Testing of the HS9-1840ARH 16-Channel Analog Multiplexer," http://radhome.gsfc.nasa.gov/ radhome/ papers/ T051010_HS91840ARH.pdf, Mar. 2010.

[42] Dakai Chen, and Anthony Phan, "Elevated Temperature Pulsed-Laser Irradiation Test Plan for the ISL28196 Voltage Comparator," Test Report, http://radhome.gsfc.nasa. gov/radhome/ papers/ NRL031610 ISL28196.pdf, Mar. 2010.

[43] Dakai Chen, and Anthony Phan, "Laser SEE Test Report for the LTC6400-20 Differential Output Amplifier/ADC Driver," http://radhome.gsfc.nasa.gov/ radhome/ papers/ NRL031610 LTC6400.pdf, Mar. 2010.

[44] Dakai Chen, and Anthony Phan, "Proton Irradiation Test Plan for the LTC6400-20 Differential ADC Driver," http://radhome.gsfc.nasa.gov/ radhome/ papers/ 1032910 LTC6400.pdf, Mar. 2010.

[45] Jean-Marie Lauenstein, Anthony Phan, Timothy Irwin, and Hak Kim, "Single event effects testing of the Vishay SiB455EDK p-type Power MOSFET," Test Report, http://radhome.gsfc. nasa.gov/ radhome/papers/ T081810_SiB455EDK.pdf, Aug. 2010.

[46] Jean-Marie Lauenstein, Dakai Chen, Anthony Phan, Timothy Irwin, and Hak Kim, "Single Event Effects Testing of the Vishay Si7431DP $200 \mathrm{~V}$ p-type Power MOSFET," Test Report, http://radhome.gsfc. nasa.gov/ radhome/papers/ LBNL012100_ LBNL032811_ Si7431DP.pdf, Mar. 2010.

[47] Jean-Marie Lauenstein, et al., "Single Event Effects Testing of the Semicoa Engineering SCF9550 450 V n-type power MOSFET," Test Report, http://radhome.gsfc. nasa.gov/ radhome/papers/ TAMU1ODEC_ SCF9550.pdf, Dec. 2010.

[48] Jean-Marie Lauenstein, et al., "Single Event Effects Testing of the Semicoa Engineering 2 N7270 $500 \mathrm{~V}$ n-type power MOSFET, Test Report, http://radhome.gsfc. nasa.gov/ radhome/papers/ TAMU10AUG_ 2N7270.pdf, Aug. 2010.

[49] Jean-Marie Lauenstein, et al., "Single Event Effects Testing of the STMicro Engineering STRH100NIOFSY3 $100 \mathrm{~V}$ n-type power MOSFET, Test Report, http://radhome.gsfc. nasa.gov/ radhome/papers/ TAMU10AUG_TAMU10DEC_STRH100N10FSY3.pdf, Aug. 2010, Dec. 2010.

[50] Jonathan Pellish, "Heavy Ion Single-Event Latchup Characterization of the Cypress STAR-250NDR Active Pixel Sensor," http://radhome.gsfc. nasa.gov/ radhome/papers/ T081610_STAR-250NDR.pdf, Aug. 2010.

To be presented by Martha O'Bryan at the International Electrical and Electronics Engineering (IEEE) Nuclear and Space Radiation Effects Conference (NSREC), July 27, 2011, Las Vegas, NV, and published on nepp.nasa.gov and radhome.gsfc.nasa.gov. 
[51] Hak S. Kim, Anthony Phan, Mark Friendlich, and Melanie Berg, "Single Event Latch Susceptibility Test of Different Circuit Areas of Numonyx Phase Changing Memory A33," http://radhome.gsfc.nasa.gov/ radhome/ papers/ T052110_PCMA33.pdf, Mar. 2010.

[52] Tim Oldham, Mark Friendlich, Hak Kim, Melanie Berg, and Kenneth A. LaBel, "HI SEE Test Report for the Numonyx 4G NAND Flash Memory," http://radhome.gsfc.nasa.gov/ radhome/ papers/ T052210 NAND04GW3B2DN6.pdf, Mar. 2010.

[53] Tim Oldham, Mark Friendlich, and Farokh lrom, "Heavy lon SEE Test Report for the Current Spike Experiment," http://radhome.gsfc.nasa.gov/ radhome/ papers/ T120710_K9F8G08U0M_MT29F4G08AAAWP.pdf, Dec. 2010.

[54] T. R. OIdham, M. R. Friendlich, and K. A. LaBel, "Investigation of Current Spike Phenomena During Heavy Ion Irradiation of NAND Flash Memory," NSREC W-25 submitted for publication in IEEE Radiation Effects Data Workshop, Jul. 2011.

[55] J. A. Pellish, M. A. Carts, S. Buchner, M. J. Campola, M. A. Xapsos, and C. M. Seidleck, "Pulsed Laser Single-Event Transient Testing of the MiniCircuits RAM-3+ MMIC InGaP HBT Amplifier," http://radhome.gsfc.nasa.gov/ radhome/ papers/ NRL0129I0_RAM3.pdf, Jan. 2010.

[56] J. A. Pellish, M. A. Carts, S. Buchner, M. J. Campola, M. A. Xapsos, and C. M. Seidleck, "Pulsed Laser Single-Event Transient Testing of the MiniCircuits RAM-6+ MMIC Silicon Amplifier," http://radhome.gsfc.nasa.gov/ radhome/ papers/ NRL012910_RAM6.pdf, Jan. 2010.

[57] J. A. Pellish, M. A. Carts, S. Buchner, M. J. Campola, M. A. Xapsos, and C. M. Seidleck, "Pulsed Laser Single-Event Transient Testing of the Hittite HMC422 MMIC GaAs Mixer," http://radhome.gsfc.nasa.gov/ radhome/ papers/NRL012910_HMC422.pdf, Jan. 2010.

[58] Timothy R. Oldham, Farokh Irom, Mark Friendlich, Duc Nguyen, Hak Kim, Melanie Berg, and Kenneth A. LaBel, "Current Spike Investigation for NAND Flash Memory," Single Event Effects (SEE) Symposium, La Jolla, CA, http://radhome.gsfc.nasa.gov/ radhome/ papers/ SEESYM11 Oldham.pdf, Apr. 2011

[59] Michael Campola, Jonathon Pellish, Anthony Phan, Hak Kim, Tony Sanders, and Mike Xapsos, "Pulsed Laser and Heavy Ion Single Event Transient (SET) Testing of the 1S705RH Power On Reset Circuit," http://radhome.gsfc.nasa.gov/ radhome/ papers/ T051010_IS705RH.pdf, May 2010.

[60] K. P. Rodbell, D. F. Heidel, M. S. Gordon, K. G. Stawiasz, J. A. Pellish, K. A. LaBel, P. W. Marshall, M. D. Berg, H. S. Kim, M. R. Friendlich, A. M. Phan, and C. M. Seidleck, "32 and $45 \mathrm{~nm}$ Radiation Hardened by Design (RHBD) SOI Latches," NSREC C3 submitted for publication in IEEE Trans. Nucl. Sci., Dec. 2011.
[61] N. Seifert, B. Gill, J. A. Pellish, K. A. LaBel, and P. W. Marshall, "On the Susceptibility of Latches to Low-Energy Protons," NSREC C4 submitted for publication in IEEE Trans. Nucl. Sci., Dec. 2011.

[62] D. F. Heidel, K. P. Rodbell, P. Oldiges, M. S. Gordon, H. H. K. Tang, E. H. Cannon, and C. Plettner, "Single-Event-Upset Critical Charge Measurements and Modeling of $65 \mathrm{~nm}$ Silicon-on-Insulator Latches and Memory Cells," IEEE Trans. Nucl. Sci., vol. 53, no. 6, pp. 3512-3517, 2006.

[63] J. A. Felix, M. R. Shaneyfelt, J. R. Schwank, S. M. Dalton, P. E. Dodd, and J. B. Witcher, "Enhanced degradation in power MOSFET devices due to heavy ion irradiation," IEEE Trans. Nucl. Sci.,, vol. 54, no. 6, pp. 2181-2189, Dec. 2007.

[64] S. Kuboyama, A. Maru, N. Ikeda, T. Hirao, and T. Tamura, "Characterization of Microdose Damage Caused by Single Heavy Ion Observed in Trench Type Power MOSFETs," IEEE Trans. Nucl. Sci., vol 57, no. 6, pp. 3257-3261, Dec. 2010. .

[65] J. S. Melinger, S. Buchner, D. McMorrow, W. J. Stapor, T. R. Weatherford, A. B. Campbell, and H. Eisen, "Critical evaluation of pulsed laser method for single-event effects testing and fundamental studies," IEEE Trans. Nucl. Sci., vol. 41, no. 6, pp. 2574-2584, Dec. 1994.

[66] D. McMorrow, J. S. Melinger, S. Buchner, T. Scott, R. D. Brown, and N. F. Haddad, "Application of a pulsed laser for evaluation and optimization of SEU-hard designs," IEEE Trans. Nucl. Sci., vol. 47, no. 3, pp. 559-565, Jun. 2000.

[67] S. Buchner, D. McMorrow, C. Poivey, J. Howard, Y. Boulghassoul, L. W. Massengill, R. Pease, and M. Savage, "Comparison of single-event transients induced in an operational amplifier (LM124) by pulsed laser light and a broad beam of heavy ions," IEEE Trans. Nucl. Sci., vol. 51, no. 5, pp. 2776-2781, Oct. 2004.

[68] F. Irom and D. Nguyen, Single event effect characterization of high density commercial NAND and NOR nonvolatile flash memories, IEEE Trans. Nucl. Sci., vol. 54, no. 6, pp.2547-2553, Dec. 2007.

[69] F. Irom, D.N. Nguyen, M. Bagatin, G. Cellere, S. Gerardin, and A. Paccagnella, Catastrophic failure in highly scaled commercial NAND flash memories, IEEE Trans. Nucl. Sci., vol. 57, no. 1, pp. 266-271, Dec. 2010. 